\title{
The Chemical Biology of the Persulfide (RSSH)/Perthiyl (RSS.) Redox Couple and Possible Role in Biological Redox Signaling
}

Christopher L. Bianco ${ }^{1}$, Tyler A. Chavez ${ }^{1}$, Victor Sosa ${ }^{2}$, Simran, S. Saund ${ }^{2}$, Q. Nhu N. Nguyen ${ }^{3}$, Dean J. Tantillo ${ }^{3}$, Andrew S. Ichimura ${ }^{4}$, John P. Toscano ${ }^{* 1}$ and Jon M. Fukuto*2

1'Department of Chemistry, Johns Hopkins University, Baltimore, Maryland, 21218, ${ }^{2}$ Department of Chemistry, Sonoma State University, Rohnert Park, California, 94928, ${ }^{3}$ Department of Chemistry, University of California, Davis, One Shield Ave., Davis, CA, 95616, ${ }^{4}$ Department of Chemistry and Biochemistry, San Francisco State University, 1600 Holloway Avenue, San Francisco, California 94132-4163.

*to whom correspondence can be addressed: John P. Toscano, jtoscano@jhu.edu, Jon M. Fukuto,fukuto@sonoma.edu,

\begin{abstract}
The recent finding that hydropersulfides (RSSH) are biologically prevalent in mammalian systems has prompted further investigation of their chemical properties in order to provide a basis for understanding their potential functions, if any. Hydropersulfides have been touted as hyper-reactive thiol-like species that possess increased nucleophilicity and reducing capabilities compared to their thiol counterparts. Herein, using persulfide generating model systems, the ability of RSSH species to act as one-electron reductants has been examined. Not unexpectedly, RSSH is relatively easily oxidized, compared to thiols, by weak oxidants to generate the perthiyl radical (RSS.). Somewhat surprisingly, however, RSS - was found to be stable in the presence of both $\mathrm{O}_{2}$ and $\mathrm{NO}$ and only appears to dimerize. Thus, the RSSH/RSS. redox couple is readily accessible under biological conditions and since dimerization of RSS. may be a rare event due to low concentrations and/or sequestration within a protein, it is speculated that the general lack of reactivity of individual RSS. species may allow this couple to be utilized as a redox component in biological systems.
\end{abstract}

\section{Keywords}

Hydropersulfides, thiols, perthiyl radicals, nitroxides, polysulfides, S-nitrosothiols 


\section{Abbreviations}

$\mathrm{RSSH}$, generic hydropersulfide; RSS·, generic perthiyl radical, $\mathrm{RSH}$, generic thiol; RSSSSR, generic dialkyl tetrasulfide; RSSSR, generic dialkyl trisulfide; RSNO, generic $S$ nitrosothiol; RSS-NO, generic S-nitrosopersulfide; RS., generic thiyl radical; deionized, DI; TEMPOL, 4-hydroxy-2,2,6,6-tetramethylpiperidine 1-oxyl; TEMPO, 2,2,6,6-tetramethylpiperidin1-yl)oxyl; Cys-SSH, cysteine hydropersulfide; GSSH, glutathione hydropersulfide; DTPA, diethylenetriamine pentaacetic acid; NAP, $\mathrm{N}$-acetyl penicillamine; MCPD, $S$-methoxycarbonyl penicillamine disulfide; MCP-SSH, $\mathrm{N}$-methoxycarbonyl penicillamine persulfide; MCP-SS-, $\mathrm{N}$ methoxycarbonyl penicillamine perthiyl radical; MbFe"I, metmyoglobin; $\mathrm{MbFe}$ ", ferrous myoglobin; $\mathrm{MbFe}^{\|} \mathrm{O}_{2}$, oxymyoglobin. 


\section{Introduction}

Hydrogen sulfide $\left(\mathrm{H}_{2} \mathrm{~S}\right)$ is an endogenously synthesized small molecule bioregulator with numerous physiological activities [1,2,3]. For example, $\mathrm{H}_{2} \mathrm{~S}$ has been reported to be involved in the etiology of atherosclerosis [4] and cancer [5], regulate transcription via NRF2/KEAP1 [6] or NF- $\square$ B [7], control vascular tone [8], inhibit phosphodiesterase activity [9], protect against neurodegenerative diseases [10] and protect from ischemia-reperfusion toxicity [11], just to name a few. Current interest in the chemical biology of $\mathrm{H}_{2} \mathrm{~S}$ has been motivated primarily by the reports of its biological activity and potential therapeutic utility. Despite the increasing recognition that $\mathrm{H}_{2} \mathrm{~S}$ is an important physiological mediator/regulator, the biochemical targets and chemical mechanism(s) associated with these actions are, for the most part, unknown.

In a biological system, $\mathrm{H}_{2} \mathrm{~S}$ can react with disulfides (RSSR), creating an equilibrium involving $\mathrm{H}_{2} \mathrm{~S}$, RSSR, thiols (RSH) and hydropersulfides (RSSH, note: this abbreviation will be used to denote all generic hydropersulfide species) (Reaction 1) [12,13].

$$
\mathrm{RSSR}+\mathrm{H}_{2} \mathrm{~S} \rightleftharpoons \mathrm{RSSH}+\mathrm{RSH}
$$

The existence of this equilibrium reaction indicates the potential for $\mathrm{RSSH}$ formation from $\mathrm{H}_{2} \mathrm{~S}$ when RSSR is present. Moreover, a pathway for $\mathrm{H}_{2} \mathrm{~S}$ biosynthesis, conversion of cystine (CysSS-Cys) to $\mathrm{H}_{2} \mathrm{~S}$ and pyruvate, involves the intermediacy of cysteine hydroperpersulfide (Cys$\mathrm{SSH}$ ) [14]. Thus, $\mathrm{H}_{2} \mathrm{~S}$ and $\mathrm{RSSH}$ are intimately linked chemically and, presumably biologically. In support of this idea, numerous recent studies indicate that RSSH are ubiquitous and highly prevalent in mammals with concentrations of glutathione hydropersulfide (GSSH) as high as 150 micromolar in mouse brain and 50 micromolar in heart and liver [15]. Also, several labs report the prevalence of numerous protein hydropersulfides [15,16,17]. Indeed, it has been speculated that $\mathrm{RSSH}$ and polysulfides $\left(\mathrm{RS}_{n} \mathrm{H}\right.$ or $\left.\mathrm{RS}_{n} \mathrm{R}, \mathrm{n}>2\right)$ are crucial biological effectors and that $\mathrm{H}_{2} \mathrm{~S}$ can serve as a marker for their presence [18]. Although $\mathrm{H}_{2} \mathrm{~S}$ is likely to have its own functions, it is becoming increasingly evident that RSSH (and derived compounds) can be important biological effectors, regulators and signaling species [for example, 19]. Thus, the chemical biology of RSSH becomes a topic of vital importance in the attempt to delineate the mechanisms by which $\mathrm{H}_{2} \mathrm{~S}$ and related species elicit their biological actions.

Recent work from this lab and others has shown that RSSH exhibit enhanced thiol-like chemistry compared to the corresponding thiol $[20,21,22]$. That is, RSSH are more nucleophilic and reducing (both one- and two-electron) compared to thiols and it is speculated that these properties may be important to their physiological utility. Of particular interest to the current 
study is the ability of RSSH to act as a one-electron reductant. One possible reason for the superior one-electron reducing capability of RSSH species versus the corresponding thiol is the presumed stability of the oxidized product, the perthiyl radical (RSS·), compared to that of a thiyl radical (RS.) (vide infra). Thus, in order to further evaluate and predict the possible physiological utility of hydropersulfides as one-electron donors or redox regulators, the formation, stability and reactivity of RSS. has been examined.

\section{Material and Methods}

Reagents and Instruments: 4-Hydroxy-2,2,6,6-tetramethylpiperidine 1-oxyl (TEMPOL), diethylenetriamine pentaacetic acid (DTPA), potassium ferricyanide $\left(\mathrm{K}_{3} \mathrm{Fe}(\mathrm{CN})_{3}\right), \mathrm{N}$-acetyl penicillamine (NAP) and myoglobin (horse heart) were purchased from Sigma-Aldrich (St. Louis, Mo). Sodium hydrogen sulfide (NaSH) was purchased from Strem Chemicals, Inc.

(Newburyport, MA). The persulfide donor S-methoxycarbonyl penicillamine disulfide (MCPD) was synthesized as previously described [23]. The $N$-methoxycarbonyl penicillamine persulfide (MCP-SSH) and N-methoxycarbonyl penicillamine perthiyl radical (MCP-SS-) were synthesized in situ as described below. S-Nitroso glutathione was also synthesized as previously described [24]. All other reagents and chemicals were purchased from commercial suppliers and were of the highest purity available.

UV-vis spectra were obtained on a Shimadzu 2501 UV-vis spectrophotometer and mass spectra obtained using a Thermo TSQ Quantum Electrospray Triple Quadrupole Mass Spectrometer controlled with XCalibur 2.1 via syringe pump.

UV-vis Analysis of the Generation of Perthiyl Radicals: The reaction between TEMPOL and the hydropersulfide donor MCPD was monitored by measuring the loss of the nitroxide absorbance at $426 \mathrm{~nm}\left(\varepsilon=13.4 \mathrm{M}^{-1} \mathrm{~cm}^{-1}\right)$ [25]. A $100 \mathrm{mM}$ stock solution of TEMPOL was prepared in $0.1 \mathrm{M}$ phosphate buffer containing $50 \mu \mathrm{M}$ DTPA (pH 7.4) and $50 \mathrm{mM}$ stock solution of MCPD was prepared in deionized (DI) water (note: MCPD does not rearrange to generate a hydropersulfide in pure water). The TEMPOL stock solution was diluted into a cuvette containing the same buffer to a final concentration of $10 \mathrm{mM}$. The UV-vis spectrum was recorded to verify the concentration of TEMPOL. Next, MCPD was added to the cuvette to a final concentration of $5 \mathrm{mM}$. UV-vis spectra were recorded every 90 seconds for thirty minutes from $250-750 \mathrm{~nm}$.

Potassium ferricyanide, $\mathrm{K}_{3} \mathrm{Fe}(\mathrm{CN})_{6}$, was also used as an oxidant and its reduction from $\mathrm{Fe}(\mathrm{III})$ to $\mathrm{Fe}(\mathrm{II})$ was monitored by measuring the loss in absorbance at $420 \mathrm{~nm}\left(\varepsilon=1000 \mathrm{M}^{-1} \mathrm{~cm}^{-}\right.$ 1) [26 Estabrook, 1961]. A $2 \mathrm{mM}$ stock solution of $\mathrm{K}_{3} \mathrm{Fe}(\mathrm{CN})_{6}$ was prepared in $0.1 \mathrm{M}$ phosphate 
buffer ( $\mathrm{pH}$ 7.4) without metal chelator and a $1 \mathrm{mM}$ stock solution of MCPD was prepared in DI water. For the reaction, $\mathrm{K}_{3} \mathrm{Fe}(\mathrm{CN})_{6}$ was diluted in a cuvette containing the same buffer to a final concentration of $200 \mu \mathrm{M}$. Before starting the reaction, a UV-vis spectrum of $\mathrm{K}_{3} \mathrm{Fe}(\mathrm{CN})_{6}$ only was measured to confirm its concentration. MCPD was then added to the cuvette to final concentration of $100 \mu \mathrm{M}$. Spectra were measured every 90 seconds for 30 minutes.

Traditionally, persulfides have been generated via nucleophilic attack of disulfides by $\mathrm{H}_{2} \mathrm{~S} / \mathrm{NaSH} / \mathrm{Na}_{2} \mathrm{~S}[13,27]$. To confirm that the persulfide donor MCPD behaves similarly to traditional persulfide generating systems, glutathione persulfide (GSSH) was generated from the reaction of $\mathrm{NaSH}$ with glutathione disulfide (GSSG) in the presence of TEMPOL. Stock solutions (50 mM) of GSSG and NaSH were prepared separately in $0.1 \mathrm{M}$ phosphate buffer with $50 \mu \mathrm{M}$ DTPA ( $\mathrm{pH}$ 7.4). A $100 \mathrm{mM}$ stock solution of TEMPOL was prepared in the same buffer. For the reaction, GSSG was diluted into a cuvette of the same buffer to a final concentration of $5 \mathrm{mM}$. Next, $\mathrm{NaSH}$ was added to a final concentration of $5 \mathrm{mM}$. The cuvette was sealed with a rubber septum and the mixture was allowed to equilibrate for 30 minutes at room temperature. TEMPOL was then added via syringe to a final concentration of $10 \mathrm{mM}$. UV-vis spectra were recorded every 90 seconds for 30 minutes.

As controls, the reactions of $N$-acetyl penicillamine (NAP), which is considered to be a relatively analogous thiol to the hydropersulfide generated from decomposition of MCPD ( $N$ methoxycarbonyl penicillamine persulfide, MCP-SSH), with both TEMPOL and $\mathrm{K}_{3} \mathrm{Fe}(\mathrm{CN})_{6}$ were also monitored using analogous conditions as those for the reaction of MCPD with TEMPOL or $\mathrm{K}_{3} \mathrm{Fe}(\mathrm{CN})_{6}$ (described above). For reactions with TEMPOL, a $50 \mathrm{mM}$ stock solution of NAP was prepared in $0.1 \mathrm{M}$ phosphate buffer with $50 \mu \mathrm{M}$ DTPA (pH 7.4). For the reaction with $\mathrm{K}_{3} \mathrm{Fe}(\mathrm{CN})_{6}$, a $1 \mathrm{mM}$ stock solution of NAP was prepared in $0.1 \mathrm{M}$ phosphate buffer $(\mathrm{pH} 7.4)$ without metal chelator. Reactions were performed analogously to those listed above for MCPD with TEMPOL and $\mathrm{K}_{3} \mathrm{Fe}(\mathrm{CN})_{6}$, respectively.

EPR Analysis for the Reduction of TEMPO by MCPD: To enhance sensitivity, EPR experiments were performed in organic solvent $(\mathrm{MeOH})$, and therefore, the organic soluble TEMPOL analog, 2,2,6,6-tetramethylpiperidin-1-yl)oxyl (TEMPO) was used. Importantly, the redox potentials for TEMPOL and TEMPO are nearly identical [28]. MCPD (5 mM) was added to $10 \mathrm{~mL}$ of methanol containing tetrabutylammonium hydroxide (10 mM) and TEMPO $(5 \mathrm{mM})$. The EPR spectra were obtained on a Bruker X-band EMX spectrometer with a gun diode as the microwave source running at $9.48 \mathrm{GHz}$. The TEMPO signal was monitored in an EPR flat cell over time for $100 \mathrm{~min}$ at room temperature. 
The Reaction of TEMPOL with Hydropersulfides - Analysis for Dioxygen $\left(\mathrm{O}_{2}\right)$

Consumption: The possible reaction of $\mathrm{N}$-methoxycarbonyl penicillamine perthiyl radical (MCPSS.) with $\mathrm{O}_{2}$ was monitored by measuring the loss of $\mathrm{O}_{2}$ from a reaction solution containing TEMPOL and MCPD using a Clark-type electrode (YSI, Ohio). A $10 \mathrm{~mL}$ two-neck round bottom flask fitted with a septum and an oxygen Clark-type electrode was filled with $15 \mathrm{~mL}$ of $0.1 \mathrm{M}$ phosphate buffer containing $50 \mu \mathrm{M}$ DTPA ( $\mathrm{pH}$ 7.4) to limit headspace. A stock solution of TEMPOL (75 mM) was prepared in the same buffer and a stock solution of MCPD (7.5 mM) was prepared in DI water. For monitoring dioxygen consumption, the electrode was first allowed to equilibrate under ambient conditions for 20 minutes. After this equilibration period, dioxygen readings were recorded every minute for the next 10 minutes. Then, 100 or $20 \mu \mathrm{L}$ of the TEMPOL stock solution was added to the flask (final [TEMPOL] $=500$ or $100 \mu \mathrm{M}$, respectively) via syringe and dioxygen readings continued to be recorded another 10 minutes. At this time, 100 or $20 \mu \mathrm{L}$ of the MCPD stock solution was added (final [MCPD] $=200$ or $10 \mu \mathrm{M}$, respectively) via syringe and recordings taken for the next 20 minutes.

The Reaction of TEMPOL with Hydropersulfides - Analysis for Nitric Oxide (NO) Consumption: The reaction of the MCP-SS. and NO was examined by monitoring the rate of loss of NO in the presence of added MCPD using an NO electrode (ISO-NO probe, WPI, Sarasota, FL). For this, a $10 \mathrm{~mL}$ two-neck round bottom flask fitted with a septum and a nitric oxide probe was filled with $15 \mathrm{~mL}$ of $0.1 \mathrm{M}$ phosphate buffer containing $50 \mu \mathrm{M}$ DTPA $(\mathrm{pH} 7.4)$ to limit headspace. The flask was purged with $\mathrm{N}_{2}$ for 30 minutes. The flask was then sparged with nitric oxide for another 15 minutes to achieve an atmosphere of nitric oxide. The probe was then allowed to equilibrate 10 minutes with stirring. Continuous nitric oxide measurements were then recorded for 15 minutes using DUO-18 software. After 15 minutes, a solution of TEMPOL previously purged with $\mathrm{N}_{2}$ in the same buffer was added via syringe to a final concentration of $2.5 \mathrm{mM}$ and recording continued for another 15 minutes. Finally, a solution of MCPD in DI water previously purged with $\mathrm{N}_{2}$ was added via syringe to a final concentration of $500 \mu \mathrm{M}$ and recordings continued for another 30 minutes.

ESI-MS Analysis of Polysulfides from Reactions of MCP-SSH with TEMPOL or Potassium Ferricyanide: The effect of the addition of oxidants to decomposing solutions of MCPD and equilibrated samples of NaSH/GSSG on the relative ratios of formed alkyl polysulfides was monitored using single ion monitoring (SIM) ESI-MS. A $50 \mathrm{mM}$ stock solution of TEMPOL was prepared in $50 \mathrm{mM}$ ammonium phosphate buffer containing $50 \mu \mathrm{M}$ DTPA (pH 7.4) and a $10 \mathrm{mM}$ stock solution of MCPD was prepared in DI water. For the reaction, $100 \mu \mathrm{L}$ of TEMPOL (final [TEMPOL] $=5 \mathrm{mM}$ ) was diluted into $800 \mu \mathrm{L}$ of the same buffer. Next, $100 \mu \mathrm{L}$ of 
the MCPD stock solution was added to the buffer mixture (final [MCPD] $=1 \mathrm{mM}$ ) and the mixture was allowed to stand at room temperature for 30 minutes. A $50 \mu \mathrm{L}$ aliquot of the reaction mixture was diluted 5 times with methanol and injected directly into a Thermo TSQ Quantum Triple Quadrupole Mass Spectrometer controlled with XCalibur 2.1 via syringe pump. Spectra were acquired in positive ion mode with a spray voltage of $3500 \mathrm{~V}$, a capillary temperature of $250{ }^{\circ} \mathrm{C}$ and a flow rate of $1 \mu \mathrm{L} / \mathrm{min}$. Ion optics were optimized for all ions of interest.

Similarly, MCP-SSH oxidation by $\mathrm{K}_{3} \mathrm{Fe}(\mathrm{CN})_{6}$ was examined. A $50 \mathrm{mM}$ stock solution of $\mathrm{K}_{3} \mathrm{Fe}(\mathrm{CN})_{6}$ was prepared in $50 \mathrm{mM}$ ammonium phosphate buffer ( $\mathrm{pH}$ 7.4) without metal chelator and a stock solution of MCPD was prepared as above. For the reaction, $\mathrm{K}_{3} \mathrm{Fe}(\mathrm{CN})_{6}$ was diluted into the same buffer to a final concentration of $5 \mathrm{mM}$. MCPD was added to a final concentration of $1 \mathrm{mM}$ and the reaction mixture was allowed to sit at room temperature for 30 minutes. A 50 $\mu \mathrm{L}$ aliquot was then diluted 5 times with methanol and injected into the ESI-MS for analysis (as above).

The NaSH/GSSG persulfide system was also analyzed. Stock solutions (10 mM) of $\mathrm{NaSH}$ and GSSG were prepared individually in $50 \mathrm{mM}$ ammonium phosphate buffer $(\mathrm{pH} 7.4)$ contining $50 \mu \mathrm{M}$ DTPA and a stock solution (50 mM) of TEMPOL was prepared in the same buffer. For the reaction, GSSG was diluted into the same buffer to a final concentration of 1 $\mathrm{mM}$. Next, NaSH was added to a final concentration of $1 \mathrm{mM}$. The mixture was capped with a rubber septum and allowed to equilibrate for 30 minutes at room temperature. TEMPOL was then added via syringe (final [TEMPOL] $=5 \mathrm{mM}$ ) and the reaction mixture was allowed to stand another 30 minutes at room temperature. A $50 \mu \mathrm{L}$ aliquot was then diluted 5 times with methanol and analyzed by ESI-MS (as above).

Reaction of MCP-SSH with GSNO: The production of NO from reactions involving MCPSSH or NAP and GSNO was monitored amperometrically (ISO-NO probe, WPI, Sarasota, FL) and compared to a standard curve constructed with $\mathrm{NO}$ gas. A $10 \mathrm{~mL}$ 2-neck round bottom flask fitted with a septum and an NO probe was filled with $15 \mathrm{~mL}$ of $0.1 \mathrm{M}$ phosphate buffer $(\mathrm{pH}$ 7.4) containing $50 \mu \mathrm{M}$ DTPA (55 mL) and covered with foil. A stock solution of MCPD (3 mM) was made in DI water and a stock solution of GSNO (15 mM) was prepared in $0.1 \mathrm{M}$ phosphate buffer ( $\mathrm{pH}$ 7.4) containing $50 \mu \mathrm{M}$ DTPA and covered with foil. All solutions were purged with $\mathrm{N}_{2}$ for 30 minutes. Reactions began by monitoring the NO-induced current of the buffer solution only for 10 minutes. Next, $100 \mu \mathrm{L}$ of the GSNO stock solution was added (final [GSNO] = 100 $\mu \mathrm{M})$ and NO measurements continued another 15 minutes. MCPD (100 $\mu \mathrm{L})$ was then added to 
a final concentration of $20 \mu \mathrm{M}$ and $\mathrm{NO}$ measurements were recorded for the following 30 minutes.

As a comparison, the thiol NAP was also examined. A stock solution (3 mM) of NAP was prepared in $0.1 \mathrm{M}$ phosphate buffer ( $\mathrm{pH}$ 7.4) containing $50 \mu \mathrm{M}$ DTPA and purged with $\mathrm{N}_{2}$ for 30 minutes. Experiments began by monitoring an $\mathrm{NO}$-induced current for 10 minutes in buffer only. GSNO was then added to a final concentration of $100 \mu \mathrm{M}$ and NO measurements continued another 15 minutes. Next, NAP was added to a final concentration of $20 \mu \mathrm{M}$ and NO measurements continued another 30 minutes.

Reduction of Ferric Myoglobin to Ferrous Myoglobin: The reduction of ferric myoglobin to ferrous myoglobin by MCP-SSH or NAP was monitored by measuring the loss in absorbance of ferric myoglobin at $408 \mathrm{~nm}\left(\varepsilon=188000 \mathrm{M}^{-1} \mathrm{~cm}^{-1}\right)$ [29]. A stock solution of ferric myoglobin was prepared by dissolving $1 \mathrm{mg}$ in $1 \mathrm{~mL}$ of DI water. The concentration of this stock solution was determined by diluting $100 \mu \mathrm{L}$ of the stock solution into $1 \mathrm{~mL}$ of $0.1 \mathrm{M}$ phosphate buffer $(\mathrm{pH}$ 7.4) and measuring the UV-vis absorbance at $408 \mathrm{~nm}$ (stock [metMb] $=73 \mu \mathrm{M}$ ). A $500 \mu \mathrm{M}$ stock solution of MCPD was prepared in DI water. For the reaction, the ferric myoglobin stock solution was diluted in a cuvette containing $0.1 \mathrm{M}$ phosphate buffer $(\mathrm{pH} 7.4)$ to a final concentration of $5 \mu \mathrm{M}$. The UV-vis spectrum was measured to verify the concentration. Next, MCPD was added to the cuvette to a final concentration of $50 \mu \mathrm{M}$ and the UV-vis spectrum recorded every 90 seconds for 30 minutes from $250-800 \mathrm{~nm}$.

As a control, the reduction of ferric myoglobin by NAP was also measured. A $500 \mu \mathrm{M}$ stock solution of NAP was prepared in $0.1 \mathrm{M}$ phosphate buffer $(\mathrm{pH} 7.4)$. For the reaction, ferric myoglobin was diluted into a cuvette of $0.1 \mathrm{M}$ phosphate buffer $(\mathrm{pH}$ 7.4) to a final concentration of $5 \mu \mathrm{M}$. Next, NAP was added to the cuvette to a final concentration of $50 \mu \mathrm{M}$ and the UV-Vis spectra collected as above.

Computational Studies: Optimization and frequency calculations for minima and transition state structures (TSSs) were performed for the gas phase and in water as noted in the text, modeled with the SMD implicit continuum solvation model [30] using the M06-2X/6$31+G(d, p)$ method [31 Zhao and Truhlar, 2008] implemented in Gaussian09 [32]. Intrinsic reaction coordinate (IRC) calculations were also performed to confirm which minima are connected to TSSs $[33,34]$.

\section{Results}


Hydropersulfide Generation: The in situ generation of hydropersulfides can be accomplished using a penicillamine-based donor species MCPD giving the hydropersulfide MCP-SSH [22,23,35] (Figure 1a).
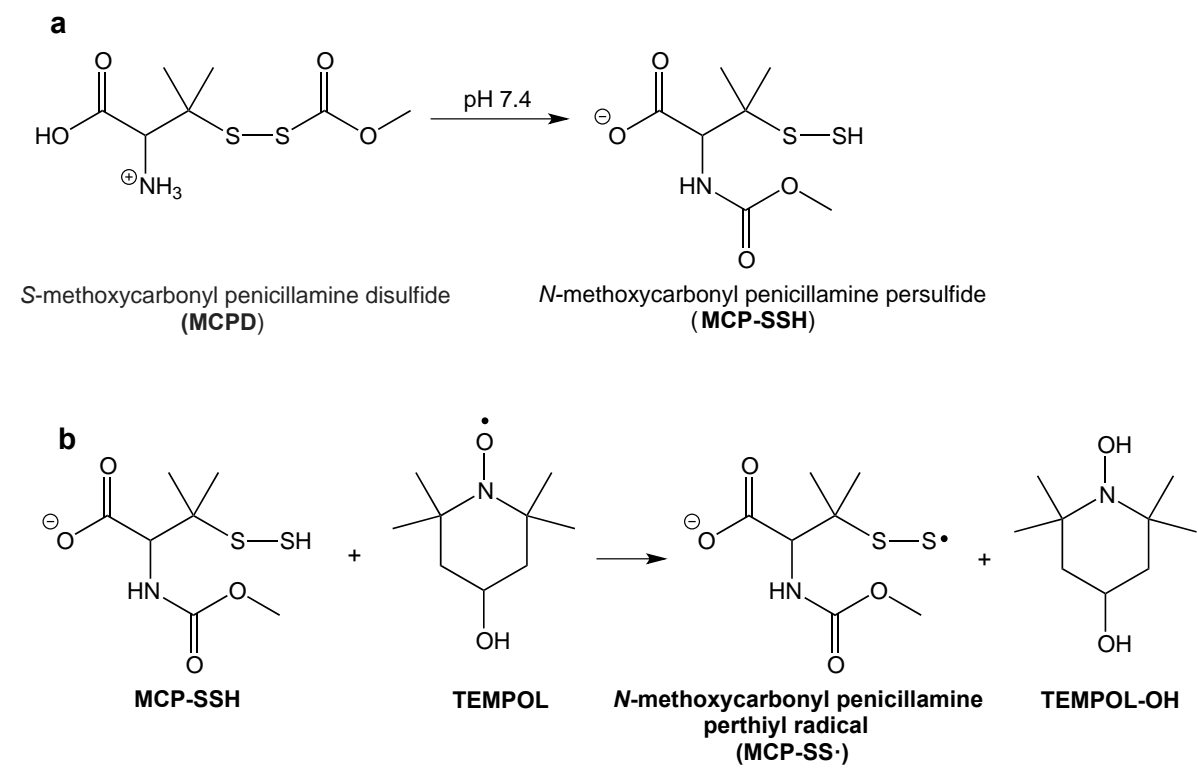

Figure 1: (a) Generation of MCP-SSH from MCPD. (b) Reduction of TEMPOL to TEMPOL-OH by MCP-SSH and generation of MCP-SS.

The reduction of TEMPOL by MCP-SSH generates the reduced TEMPOL-OH species (vide infra) and the corresponding perthiyl radical $N$-methoxycarbonyl penicillamine perthiyl radical (MCP-SS.) (Figure 1b). Many of the experiments described herein involve the use of MCPD to make MCP-SSH, which can be oxidized by TEMPOL to give MCP-SS.

Reduction of TEMPOL by Hydropersulfides: The one-electron oxidation of a persulfide was initially investigated by monitoring the reduction of TEMPOL to the corresponding hydroxylamine (Reaction 2). Importantly, TEMPOL absorbs at $426 \mathrm{~nm}$ while TEMPOL-OH has no absorbance in the visible region. As can be seen in Figure $\mathbf{2 a}$, addition of $5 \mathrm{mM}$ MCPD to a solution of $10 \mathrm{mM}$ TEMPOL causes subsequent decreases in the absorbance at $426 \mathrm{~nm}$ (TEMPOL) over 30 minutes (it should be noted that while the UV-vis spectra indicate a quantitative reduction of TEMPOL, precise quantification is difficult because other intermediate species also absorb in this region). This change in absorbance is indicative of the reduction of TEMPOL to the corresponding TEMPOL-OH [25] by MCP-SSH (Figure 1b, Reaction 3). 


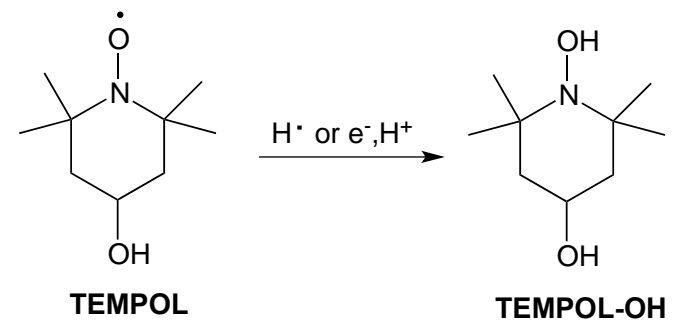

$$
\mathrm{RSSH}+\mathrm{TEMPOL} \rightarrow \mathrm{RSS} \cdot+\mathrm{TEMPOL}-\mathrm{OH}
$$

In contrast, addition ( $5 \mathrm{mM}$ ) of the analogous thiol NAP to a $10 \mathrm{mM}$ solution of TEMPOL (Figure 2a, inset) does not induce any spectral changes over a 30-minute period. This indicates that while RSSH does reduce TEMPOL to TEMPOL-OH, the corresponding RSH does not. Additionally, when $200 \mu \mathrm{M} \mathrm{K}{ }_{3} \mathrm{Fe}(\mathrm{CN})_{6}$ was used as an oxidant (instead of TEMPOL) in the presence of $100 \mu \mathrm{M}$ MCPD, a decrease in absorbance at $420 \mathrm{~nm}$ (corresponding to $\mathrm{K}_{3} \mathrm{Fe}(\mathrm{CN})_{6}$ ) was observed, presumably indicating reduction of $\mathrm{Fe}(\mathrm{III})$ to $\mathrm{Fe}(\mathrm{II})$ [36] by MCP-SSH (Figure 2b). NAP also reduced $\mathrm{K}_{3} \mathrm{Fe}(\mathrm{CN})_{6}$ however, reduction was less than $50 \%$ of that witnessed with MCP-SSH (data not shown) again consistent with the idea that persulfides are better oneelectron reductants than the corresponding thiol species. 

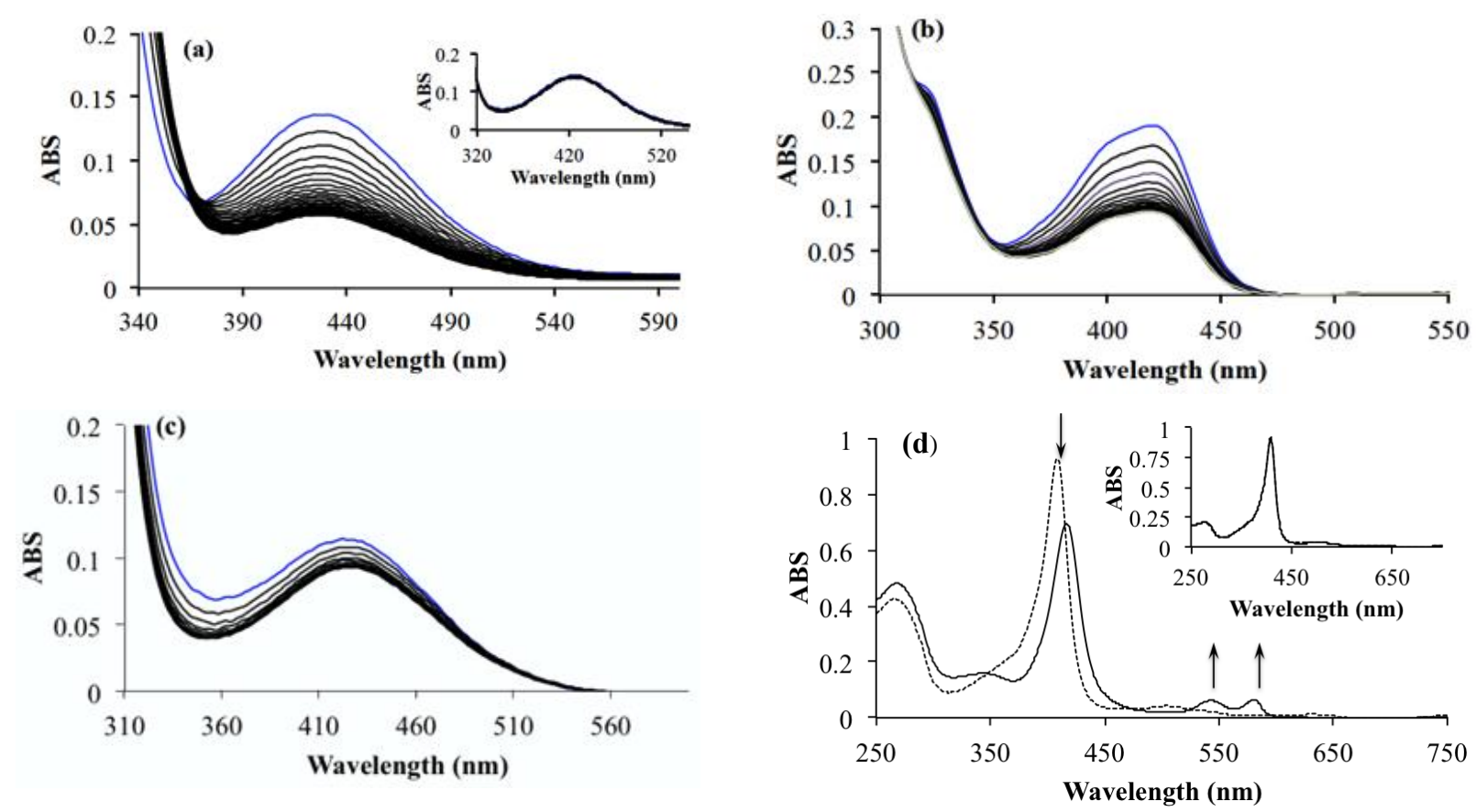

(e)
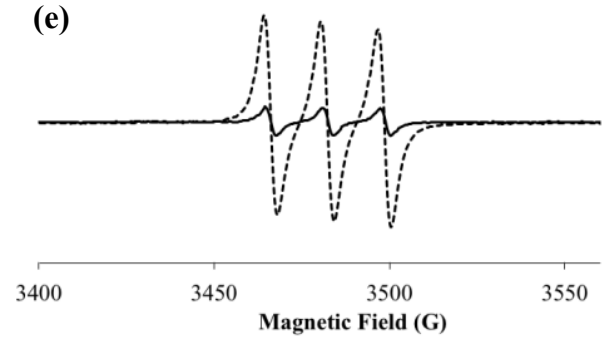

Figure 2. Spectroscopic analysis of RSSH oxidation. (a) $5 \mathrm{mM}$ MCPD and $10 \mathrm{mM}$ TEMPOL in $0.1 \mathrm{M}$ phosphate buffer containing $50 \mu \mathrm{M}$ DTPA at pH 7.4 over a 30 minute period. The loss in absorbance at $426 \mathrm{~nm}$ corresponds to the reduction of TEMPOL to TEMPOL-OH. Inset: Reaction of $5 \mathrm{mM} \mathrm{NAP}$ and $10 \mathrm{mM}$ TEMPOL. (b) Reduction of $10 \mathrm{mM} \mathrm{K}_{3} \mathrm{Fe}(\mathrm{CN})_{6}$ by 5 $\mathrm{mM}$ MCPD in $0.1 \mathrm{M}$ phosphate buffer at $\mathrm{pH} 7.4$ over a 30 minute period. The loss in absorbance at $420 \mathrm{~nm}$ corresponds to the reduction of the $\mathrm{Fe}(\mathrm{III})$ center to $\mathrm{Fe}(\mathrm{II})$. (c) Reduction of $10 \mathrm{mM}$ TEMPOL in the presence of $5 \mathrm{mM} \mathrm{NaSH}$ and $5 \mathrm{mM} \mathrm{GSSG}$ in $0.1 \mathrm{M}$ phosphate buffer containing $50 \mu \mathrm{M}$ DTPA at pH 7.4 over a 30 minute period. The loss in absorbance at $426 \mathrm{~nm}$ corresponds to the reduction of TEMPOL to TEMPOL-OH (presumably via oxidation of GSSH). (d) UV-vis spectra for various redox states of myoglobin. Dashed line: The UV-vis spectrum of $5 \mu \mathrm{M} \mathrm{MbFe}$ ll alone $(\lambda=408,502$ and $530 \mathrm{~nm})$ in $100 \mathrm{mM}$ phosphate buffer ( $\mathrm{pH}$ 7.4). Solid line: The UV-Vis spectrum that results after $5 \mu \mathrm{M} \mathrm{MbFe}{ }^{\text {III }}$ is reduced by $50 \mu \mathrm{M}$ MCPD for 30 minutes in $100 \mathrm{mM}$ phosphate buffer (pH 7.4). The increase in absorbance at $\lambda=542$ and $580 \mathrm{~nm}$ corresponds to the reduction of the MbFe $e^{\text {III }}$ center to $\mathrm{MbFe}^{\prime \prime}$ and subsequent binding of $\mathrm{O}_{2}$ to give $\mathrm{MbFe} \mathrm{O}_{2}$. Inset: UV-vis spectra of both $5 \mu \mathrm{M} \mathrm{MbFe} e^{\text {III }}$ and $5 \mu \mathrm{M} \mathrm{MbFe} e^{\text {III }}$ treated with $50 \mu \mathrm{M}$ NAP for 30 minutes in $100 \mathrm{mM}$ phosphate buffer ( $\mathrm{pH}$ 7.4). No spectral changes are observed after the addition of NAP to the solution of MbFe ${ }^{\prime l l}$ in this case. (e) EPR spectra for the reduction of TEMPO by MCP-SSH. Dashed line: EPR spectrum of $5 \mathrm{mM}$ TEMPO in methanol. Solid line: Resulting spectrum after a 30 minute incubation of $5 \mathrm{mM}$ TEMPO with $5 \mathrm{mM}$ MCPD in the presence of $10 \mathrm{mM}$ tetrabutylammonium hydroxide in methanol. The loss in signal intensity corresponds to the reduction of TEMPO to TEMPO-OH by MCP-SSH. 
To confirm that the MCP-SSH donor, MCPD, displays similar chemistry to that of a traditional persulfide-generating system (Reaction 4), the reaction of TEMPOL with GSSG/NaSH was also examined.

$$
\mathrm{GSSG}+\mathrm{H}_{2} \mathrm{~S} \rightleftharpoons \mathrm{GSSH}+\mathrm{GSH}
$$

Addition of $10 \mathrm{mM}$ TEMPOL to an equilibrated solution of $5 \mathrm{mM} \mathrm{NaSH}$ and GSSG gave similar results (Figure 2c) to those found with MCPD (Figure 2a). The NaSH/GSSG system caused a decrease in the absorbance at $426 \mathrm{~nm}$ (TEMPOL), though to a lesser extent (presumably due to low GSSH concentrations as a result of the equilibrium of Reaction $\mathbf{4}$ favoring the left side of the reaction), again indicating the reduction of TEMPOL to TEMPOL-OH. It should be noted that the active reductant in this system is presumably GSSH since no reduction of TEMPOL was observed in the presence of GSSG or GSH alone. Minor reduction of TEMPOL was observed after the addition of NaHS only. However, reduction of TEMPOL was significantly decreased when NaHS was pretreated with the phosphine TCEP, which reduces contaminating polysulfur species [21], thus indicating these contaminants were primarily responsible for NaHS-induced reduction of TEMPOL (data not shown).

Reduction of Ferric Myoglobin to Ferrous Myoglobin by MCP-SSH: Thus far it appears that hydropersulfides (e.g. MCP-SSH) are good reducing agents, presumably generating the corresponding perthiyl radical, since relatively weak oxidants such as TEMPOL and $\mathrm{K}_{3} \mathrm{Fe}(\mathrm{CN})_{6}$ are capable of performing this oxidation. In order to begin to evaluate the possible biological relevance of this chemistry, the reaction of MCP-SSH with the ferric hemeprotein metmyoglobin (MbFe $\left.{ }^{\prime \prime \prime}\right)$ was examined. As shown in Figure 2d, when $5 \mu \mathrm{M} \mathrm{MbFe}{ }^{I I I}(\lambda=408,502$ and $530 \mathrm{~nm}$, dashed line) is treated with $50 \mu \mathrm{M}$ MCPD (under aerobic conditions that will generate MCP$\mathrm{SSH}$ ), it is reduced to the ferrous myoglobin species (MbFe"), which is seen as the ferrous $-\mathrm{O}_{2}$ complex, oxymyoglobin ( $\mathrm{MbFe}^{\mathrm{I}} \mathrm{O}_{2}, \lambda=542$ and $580 \mathrm{~nm}$, solid line) after 30 minutes. In contrast, when $5 \mu \mathrm{M} \mathrm{MbFe} \mathrm{e}^{\text {III }}$ is treated with $50 \mu \mathrm{M}$ of the analogous thiol NAP, no apparent reduction of MbFe ${ }^{\text {III }}$ is observed (Figure 2d, inset), consistent with the idea that persulfide species are superior one-electron reductants compared to the corresponding thiol.

EPR Analysis for the Reduction of TEMPO by MCPD: The UV-vis data presented above indicates that persulfides are capable of reducing nitroxides and appear to be better oneelectron reductants compared to the corresponding thiol. To confirm the reduction of nitroxides by MCP-SSH further, EPR analysis of the reduction of the TEMPOL analog TEMPO $(2,2,6,6-$ tetramethylpiperidine 1-oxyl, which differs only by absence of a non-reactive hydroxyl group) 
was performed. The spectrum of Figure $2 \mathrm{e}$ displays the EPR signal of $5 \mathrm{mM}$ TEMPO in methanol (dashed line). A typical triplet full line spectrum for the nitroxide is observed. Incubation of $5 \mathrm{mM}$ TEMPO with $5 \mathrm{mM}$ MCPD and $10 \mathrm{mM}$ tetrabutylammonium hydroxide for 30 minutes causes a loss in signal intensity of the TEMPO (solid line). It should be noted that this reaction was performed in methanol and therefore MCPD requires the addition of base (tetrabutylammonium hydroxide) in order to form MCP-SSH. Consistent with the UV-vis data presented above for TEMPOL, these EPR data imply that MCP-SSH is a good one-electron reductant, capable of reducing TEMPO nitroxide to the corresponding hydroxylamine TEMPO$\mathrm{OH}$. It is important to mention that the paramagnetic perthiyl radical species was not observed by EPR, likely due to the favorable dimerization of the perthiyl to the tetrasulfide (vide infra). Moreover, attempted trapping of an intermediate MCP-SS. species using 5,5-dimethyl-1pyrroline-N-oxide (DMPO) was unsuccessful. This is likely due to: 1) the perthiyl radical being more stable than the DMPO nitroxide adduct that would be formed upon reaction of RSS. with DMPO and therefore, RSS - dimerization would dominate, giving the diamagnetic tetrasulfide species or, 2) the trapping reaction was highly reversible and dimerization of the perthiyl radical to give the diamagnetic tetrasulfide was again the dominant process.

Computational Evaluation of the TEMPOL-Hydropersulfide Reaction: The results above indicate that TEMPOL is capable of oxidizing MCP-SSH, generating the corresponding TEMPOL-OH and, presumably, a perthiyl radical. Using methyl hydropersulfide $\left(\mathrm{CH}_{3} \mathrm{SSH}\right)$ as a model compound, the reaction of a model RSSH and TEMPOL was examined computationally. As shown in Figure 3a, the reduction of TEMPOL by $\mathrm{CH}_{3} \mathrm{SSH}$ to give the corresponding TEMPOL-OH and $\mathrm{CH}_{3} \mathrm{SS}$. radical is slightly endergonic by about $4.6 \mathrm{kcal} / \mathrm{mol}$ with a transition state energy of about $12.8 \mathrm{kcal} / \mathrm{mol}$ (in water). Although this reaction is slightly uphill, the dimerization of the incipient $\mathrm{CH}_{3} \mathrm{SS}$. to give the tetrasulfide is $20.3 \mathrm{kcal} / \mathrm{mol}$ downhill (Figure $3 \mathbf{b}$ ) and thus able to drive the TEMPOL/alkyl hydropersulfide reaction. These calculations are consistent with the experimental finding of TEMPOL-mediated oxidation of MCP-SSH to give TEMPOL-OH and, presumably the MCP-SS., which can dimerize to the tetrasulfide. As will be shown later, the tetrasulfide is indeed the primary product of this reaction. 
a

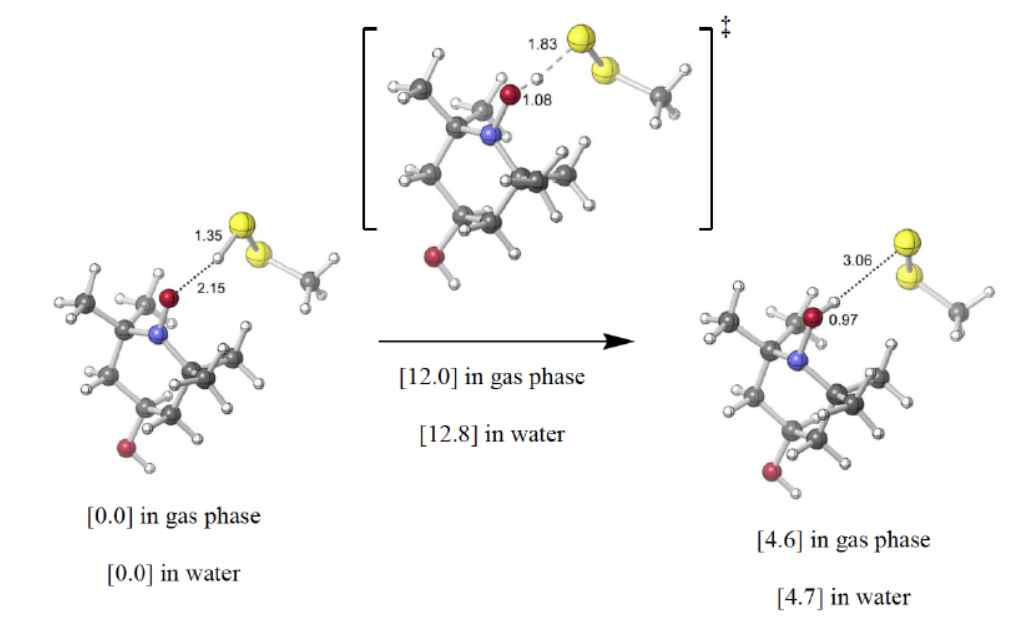

b

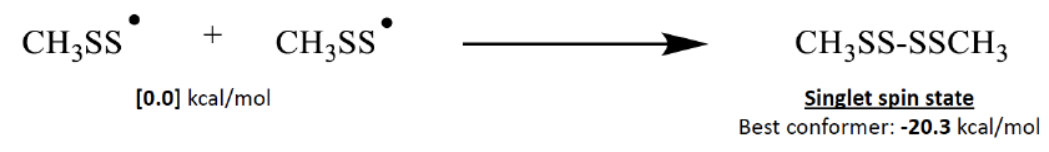

Figure 3: Computational evaluation of (a) the reduction of TEMPOL by an alkyl hydropersulfide $\left(\mathrm{CH}_{3}-\mathrm{SSH}\right)$ to give TEMPOL-OH and perthiyl radical, and (b) the thermodynamics of the dimerization of perthiyl radical $\left(\mathrm{CH}_{3}-\mathrm{SS} \cdot\right)$

ESI-MS Analysis of Polysulfides from the MCP-SSH Reactions with TEMPOL and

Potassium Ferricyanide: In the absence of any other reactants, the primary fate of the persulfide MCP-SSH generated from MCPD is formation of the dialkyl trisulfide species (MCPSSS-MCP). The fact that the trisulfide species is the predominant product is most likely due to reaction of the nucleophilic persulfide with the unreacted electrophilic precursor, MCPD, giving trisulfide (Figure 4a). However, if MCP-SSH is oxidized to MCP-SS - prior to possible reaction with the precursor MCPD, dimerization of the perthiyl species would lead to predominant dialkyl tetrasulfide generation (MCP-SSSS-MCP) (Figure 4b). 

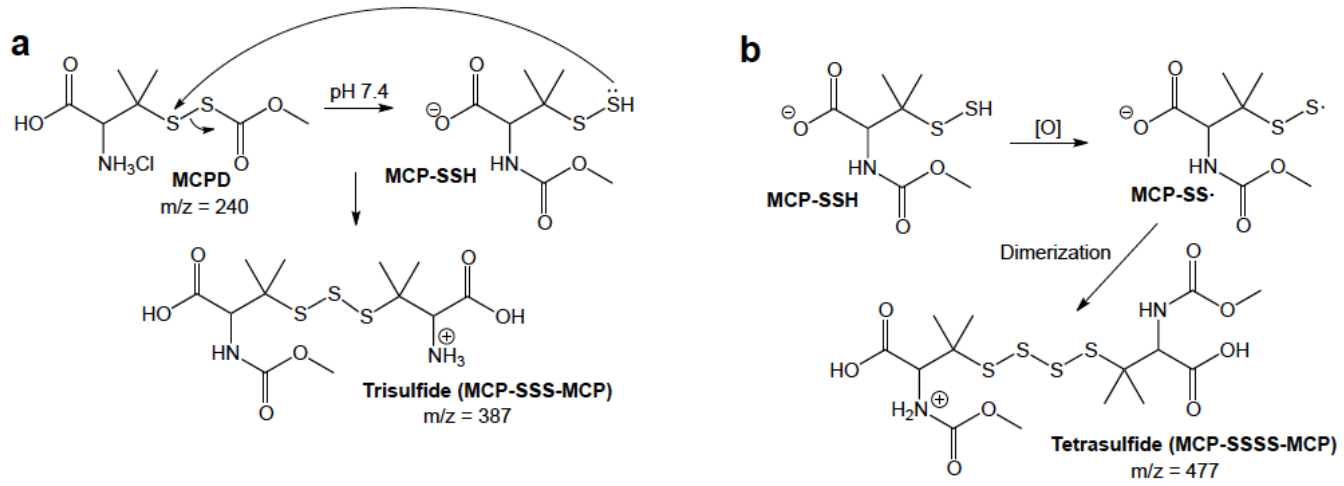

Figure 4: (a) Mechanism of MCP-SSS-MCP formation from decomposition of MCPD to MCP-SSH. (b) Formation of tetrasulfide MCP-SSSS-MCP from one-electron oxidation of MCP$\mathrm{SSH}$.

Therefore, in the presence of one-electron oxidants, the polysulfide ratios resulting from MCPD decomposition should shift from favoring the dialkyl trisulfide to favoring the dialkyl tetrasulfide (product of perthiyl radical dimerization). Using electrospray ionization - quadrupole mass spectrometry and select ion monitoring for the dialkyl trisulfide $(\mathrm{m} / \mathrm{z}=387.27)$ and the dialkyl tetrasulfide $\left(\mathrm{m} / \mathrm{z}=494.28\right.$, resulting from MCP-SSSS-MCP. $\mathrm{NH}_{4}{ }^{+}$), the ratios of these two products were qualitatively assessed under varying conditions (Figure 5). When $1 \mathrm{mM}$ MCPD is decomposed in ammonium phosphate buffer only, the predominant product is the trisulfide, which is in greater abundance compared to the tetrasulfide species (Figure 5a). In comparison, decomposition of $1 \mathrm{mM}$ MCPD in the presence of $5 \mathrm{mM}$ TEMPOL causes a change in the relative abundances of the trisulfide and tetrasulfide, now favoring the tetrasulfide (Figure $5 \mathbf{b}$ ). Similarly, use of $5 \mathrm{mM} \mathrm{K}_{3} \mathrm{Fe}(\mathrm{CN})_{6}$ as an alternative oxidant (Figure $5 \mathrm{c}$ ) also changes the ratio of alkyl polysulfides again favoring formation of the tetrasulfide over the trisulfide. Taken altogether it appears that one-electron oxidation of persulfides by numerous oxidants causes an increase in the formation of corresponding dialkyl tetrasulfides, consistent with the idea of facile oxidation of MCP-SSH to MCP-SS and subsequent dimerization. Interestingly, two-electron oxidation of a hydropersulfide by, for example, peracetic acid should give a perthiosulfenic acid (RSSOH) which can also lead to tetrasulfide formation via the reaction of an unreacted hydropersulfide with RSSOH to give tetrasulfide. Indeed, this was found to be the case as 1 $\mathrm{mM}$ MCPD reacted with $5 \mathrm{mM}$ peracetic acid at $\mathrm{pH} 7.4$ led to a relative increase in the amount of tetrasulfide product versus trisulfide (data not shown and qualitatively similar to Figures $\mathbf{5 b}$ and $\mathbf{5 c}$ ). However, the two-electron oxidation chemistry is much more complex compared to the 
one-electron oxidation chemistry as many other further oxidized products were also found in the case of two-electron oxidation (and not seen when a one-electron oxidant is used). However, since TEMPOL (as well as the other one-electron oxidants used herein) are not expected to oxidize MCP-SS - further, tetrasulfide formation via one- versus two-electron oxidation processes are considered to be mechanistically distinct.

(a)

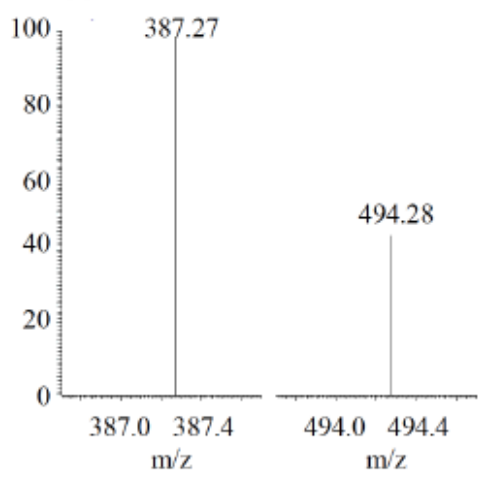

(b)

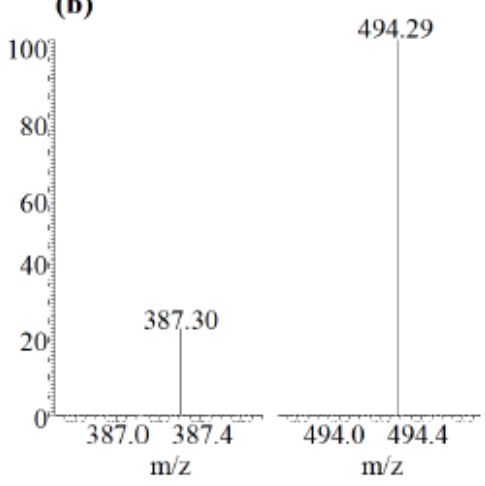

(c)

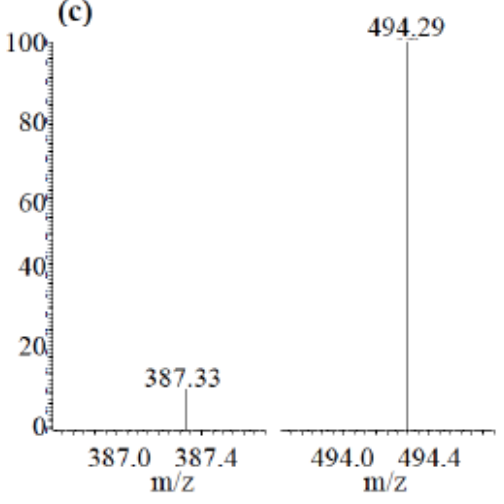

Figure 5. ESI-MS spectra displaying the ratio of sulfur species in the presence or absence of oxidizing species. (a) Relative abundance of dialkyl trisulfide (MCP-SSS-MCP, $\mathrm{m} / \mathrm{z}=387.27$ ) and dialkyl tetrasulfide (MCP-SSSS-MCP. $\mathrm{NH}_{4}{ }^{+}, \mathrm{m} / \mathrm{z}=494.28$ ) resulting from decomposition of MCPD alone in $50 \mathrm{mM}$ ammonium phosphate buffer (pH 7.4) containing $50 \mu M$ DTPA. (b) Shift in trisulfid/tetrasulfide product ratio when 1mM MCPD is decomposed in the presence of $5 \mathrm{mM}$ TEMPOL in $50 \mathrm{mM}$ ammonium phosphate buffer ( $\mathrm{pH} 7.4$ ) containing $50 \mu M$ DTPA. (c) Shift in trisulfide versus tetrasulfide ratio when $1 \mathrm{mM} \mathrm{MCPD}$ is decomposed in the presence of $5 \mathrm{mM}$ $\mathrm{K}_{3} \mathrm{Fe}(\mathrm{CN})_{6}$ in $50 \mathrm{mM}$ ammonium phosphate buffer (pH 7.4).

\section{The Reaction of MCP-SSH with TEMPOL and Possible $\mathrm{O}_{2}$ Consumption: Thus far all} results indicate that MCP-SSH is a good one-electron reductant, resulting in MCP-SS.

formation. A possible fate of this perthiyl radical is clearly dimerization but reaction with another paramagnetic species $\mathrm{O}_{2}$ appears possible. Indeed, it has been previously reported that perthiyl radicals react with $\mathrm{O}_{2}$ [37]. Therefore, it may be expected that a reaction that generates a perthiyl radical will react with and consume $\mathrm{O}_{2}$. As shown in Figure 6a, TEMPOL expectedly does not react with $\mathrm{O}_{2}$ (i.e. no change in the level of $\mathrm{O}_{2}$ is observed in the presence of TEMPOL only). When MCPD, which decomposes to MCP-SS. in the presence of TEMPOL (Figure 1a) is added there is no consumption of $\mathrm{O}_{2}$ observed (Figure 6a, solid line). As a positive control, $\mathrm{O}_{2}$ addition to a completely degassed solution shows an increase in soluble $\mathrm{O}_{2}$ (Figure 6a, dashed line) indicating that changes in $\mathrm{O}_{2}$ levels can be observed easily. These results are consistent with a lack of reactivity of a perthiyl radical with $\mathrm{O}_{2}$. 
(a)

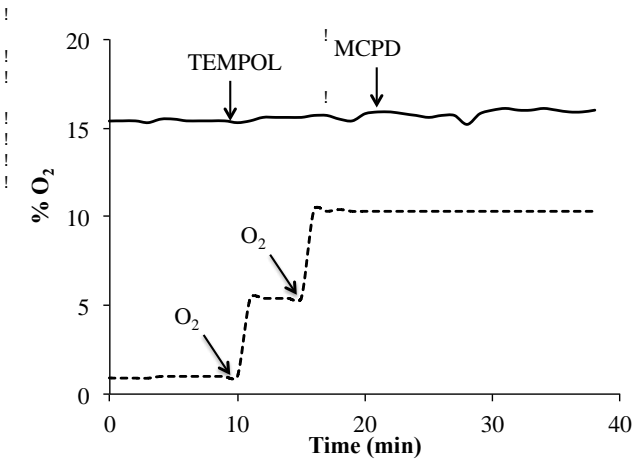

(b)

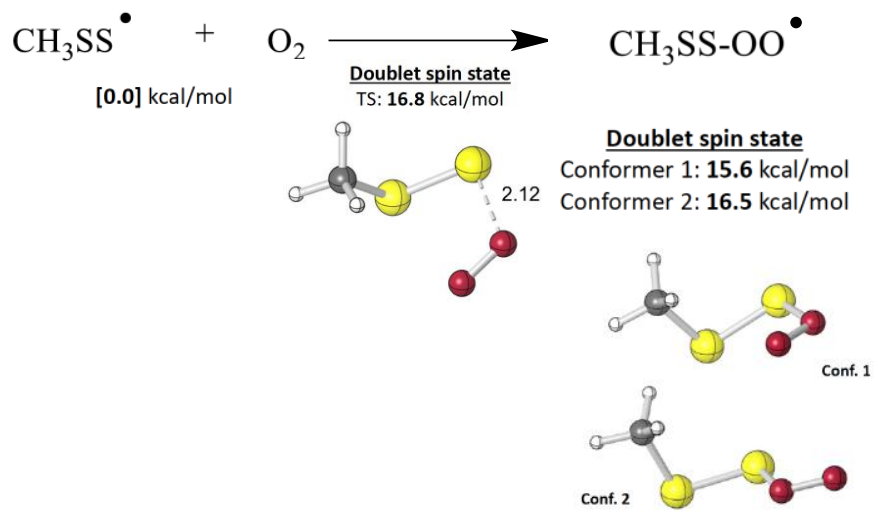

Figure 6. Analysis of the reaction of RSS. with $\mathrm{O}_{2}$. (a) Rate of dioxygen consumption detected by a Clark-type electrode. Solid line: Dioxygen consumption under ambient conditions (0-10 min), in the presence of $100 \mu \mathrm{M}$ TEMPOL (10-20 min) and in the presence of $100 \mu \mathrm{M}$ TEMPOL and $10 \mu \mathrm{M}$ MCPD (20-40 min) in $100 \mathrm{mM}$ phosphate buffer $(\mathrm{pH}$ 7.4) containing $50 \mu \mathrm{M}$ DTPA. Dashed line: Positive control for the detection of dioxygen in an $\mathrm{N}_{2}$ purged flask (0-10 $\mathrm{min})$, after the addition of $1 \mathrm{~mL}$ pure $\mathrm{O}_{2}$ gas (10-15 min) and after a second addition of $1 \mathrm{~mL}$ pure $\mathrm{O}_{2}$ gas (15-40 min). (b) Computational evaluation of the reaction of a perthiyl radical $\left(\mathrm{CH}_{3}{ }^{-}\right.$ SS. as a model compound) with $\mathrm{O}_{2}$.

Computational Assessment of the Possible Reaction of a Perthiyl Radical with $\mathrm{O}_{2}$ : The above results are consistent with the idea that RSS. species do not react with $\mathrm{O}_{2}$. This reaction was also assessed computationally. As shown in Figure 6b, the radical coupling of methyl perthiyl radical $\left(\mathrm{CH}_{3} \mathrm{SS} \cdot\right.$, a model compound) with $\mathrm{O}_{2}$ to give a peroxyl radical species $\left(\mathrm{CH}_{3} \mathrm{SS}\right.$ O-O.) in the gas-phase is thermodynamically uphill by about $16 \mathrm{kcal} / \mathrm{mol}$, consistent with the idea that RSS. species are stable radicals and reaction with $\mathrm{O}_{2}$ is unfavorable.

The Reaction of MCP-SSH with TEMPOL and Possible NO Consumption: As indicated above, the MCP-SS species resulting from the oxidation of MCP-SSH by TEMPOL does not appear to react with $\mathrm{O}_{2}$ under the current conditions. It therefore became of interest to determine if other biologically relevant paramagnetic species can react with perthiyl radicals. Thus, NO was chosen as a potential trap for MCP-SS. The trace in Figure 7a displays the rate of decay of NO, monitored using an NO-electrode, in the presence of $2.5 \mathrm{mM}$ TEMPOL and 500 $\mu \mathrm{M}$ MCPD under saturated NO conditions. 
(a)

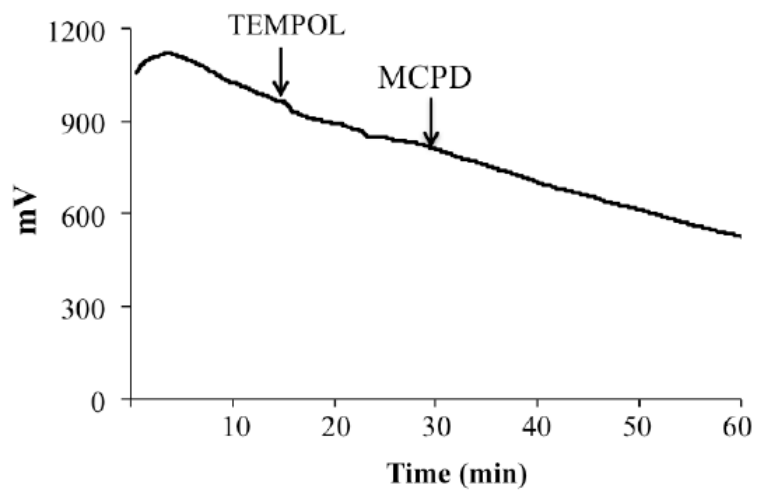

(c)

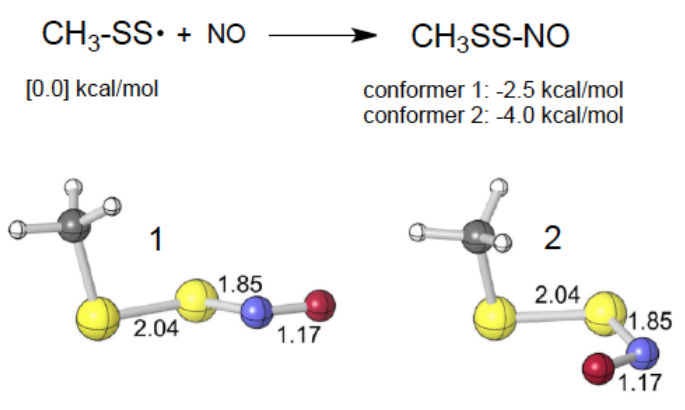

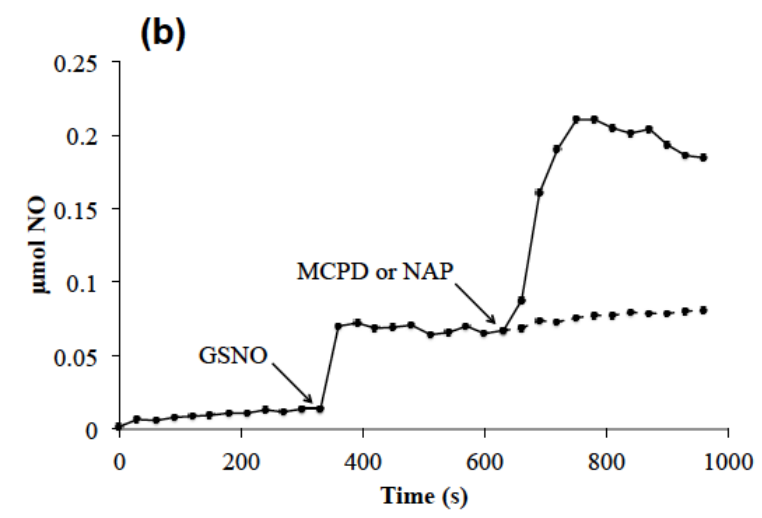

Figure 7. Analysis of the reaction of NO with RSS. (a) Amperometric detection of NO consumption. The trace shows the rate of decay of an NO-induced current in a pure NO purged flask containing $100 \mathrm{mM}$ phosphate buffer ( $\mathrm{pH}$ 7.4) containing $50 \mu \mathrm{M}$ DTPA (0-15 min), after the addition of $2.5 \mathrm{mM}$ TEMPOL (15-30 min) and after subsequent addition of $500 \mu \mathrm{M}$ MCPD. (b) Amperometric measurements for the detection of NO resulting from reactions of GSNO with MCP-SSH or NAP. All measurements were performed anaerobically in $0.1 \mathrm{M}$ phosphate buffer ( $\mathrm{pH}$ 7.4) with $50 \mu \mathrm{M}$ DTPA. The buffered solutions were allowed to equilibrate for 10 minutes prior to the addition of $100 \mu \mathrm{M}$ GSNO. Dashed line: After NAP addition. (c) Computational evaluation of the reaction of a model alkyl perthiyl radical species $\left(\mathrm{CH}_{3}-\mathrm{SS} \cdot\right)$ and NO.

As indicated by the arrows, addition of TEMPOL to the saturated buffered solution of NO does not change the natural rate of decay of the NO signal (note: NO decay in the absence of exogenous reactants is likely due to reaction with trace amounts of molecular oxygen present in the reaction mixture and slow diffusion from the experimental vessel through the septum). Subsequent addition of MCPD (under conditions whereby MCP-SS. would be generated, Figure $\mathbf{1} \mathbf{a}, \mathbf{b})$ to the solution also does not change the rate of decay of the NO signal over a 30 minute period. These data indicate that in addition to a lack of reactivity towards $\mathrm{O}_{2}, \mathrm{MCP}$-SS. also does not appear to react with NO to a significant extent under these conditions. 
Reaction of MCP-SSH with GSNO: The above results allude to a lack of reactivity of MCP-SS with NO, possibly due to a weak S-NO bond in a putative MCP-SS-NO; a result of highly stable products from bond scission (NO and MCP-SS.). To test this idea further, the generation of MCP-SS-NO was attempted using a different method. It is known that persulfides are strong nucleophiles [21] and that $S$-nitrosothiols, such as $S$-nitrosoglutathione, are electrophilic and capable of transnitrosation chemistry [for example, 38]. Thus an MCP-SS-NO species could potentially be made via Reaction $\mathbf{5}$ and if indeed the MCP-SS-NO species is unstable with respect to homolytic dissociation to give MCP-SS. and NO (Reaction 6), then it would be expected that this reaction would facilitate the release of NO from GSNO.

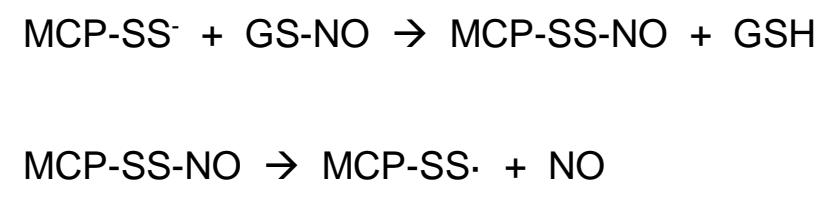

As shown in Figure $\mathbf{7 b}$, when GSNO $(100 \mu \mathrm{M})$ is added to a buffered solution, a small amount of $\mathrm{NO}$ is released (likely due to trace metals in the buffer) and the release subsides quickly giving a stable solution. However, when MCPD $(20 \mu \mathrm{M})$ is subsequently added (generating MCP-SSH), a large release of $\mathrm{NO}$ is observed (Figure $\mathbf{7 b}$, solid-dot line), consistent with Reactions 5 and 6 . However, when an analogous thiol, NAP $(20 \mu \mathrm{M})$, is added, no additional release of $\mathrm{NO}$ is observed (Figure $\mathbf{7 b}$, dashed-dot line). These results further support the idea that an S-nitrosoalkyl persulfide (e.g. MCP-SS-NO) is unstable and quickly degrades to give two relatively stable radical species, RSS. and NO.

Computational Evaluation of the Reaction of a Perthiyl Radical with NO: The above results are consistent with the idea that perthiyl radicals do not react significantly with NO. This reaction was also evaluated computationally. As shown in Figure 7c, the reaction of a perthiyl radical with $\mathrm{NO}$ is thermodynamically downhill by about $4 \mathrm{kcal} / \mathrm{mol}$ indicating a very weak interaction. Therefore, if formed, reversal of RSS-NO, yielding NO and RSS is probable at room temperature and is likely driven by RSS dimerization to the alkyltetrasulfide, a process that is thermodynamically favored (Figure $\mathbf{3 b}$ ).

\section{Discussion}

All of the results presented herein are consistent with the idea that RSSH species are good one-electron reductants. The model alkyl hydropersulfide used in this study, MCP-SSH (generated from decomposition of MCPD) (Figure 1a), was easily oxidized by the relatively 
weak one-electron oxidants TEMPOL, TEMPO, $\mathrm{K}_{3} \mathrm{Fe}(\mathrm{CN})_{6}$ and $\mathrm{MbFe}$ III' (Figure 2), a reaction that is likely driven by dimerization of the intermediate RSS. species (Figure 3). EPR results are also consistent with the idea that reaction of RSSH with TEMPO leads to the reduction of TEMPO to TEMPO-OH and, presumably, an RSS. species (Figure 2). The formation of an RSS. intermediate is further supported by the finding that RSSH oxidation leads to tetrasulfides (RSSSSR) species that are not predominant products in the absence of one-electron oxidants (Figure 5). Importantly, RSSH are also significantly more reducing compared to the corresponding thiol in that many of the reduction reactions performed by the alkyl hydropersulfides were not observed with thiols under analogous conditions (e.g. the reduction of TEMPOL or MbFe'"I, Figure 2). The reducing capabilities of alkyl hydropersulfides are not necessarily unexpected since previous work reports perthiols as efficient antioxidants capable of reacting with and quenching oxidizing radical species [39,40]. Clearly, the reducing ability of an RSSH species is at least partially due to the inherent stability of the perthiyl radical, RSS., which can be explained by the fact that the unpaired electron is resonance stabilized, a stabilizing affect unavailable to the unpaired electron on a thiyl radical, RS. [18]. The resonance stability of the perthiyl unpaired electron is analogous to the stabilization of paramagnetic nitroxides, $\mathrm{R}_{2} \mathrm{NO}$. (Figure 8) [41].

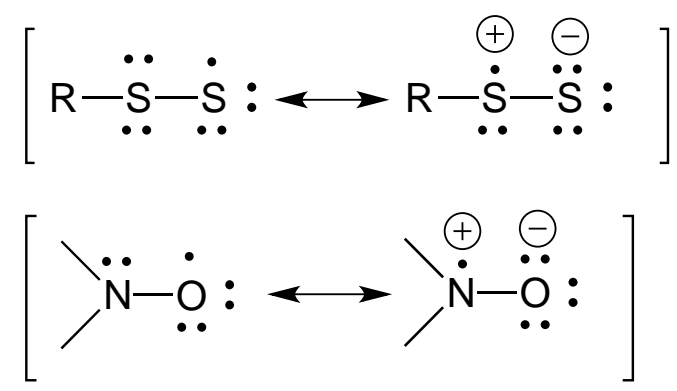

Figure 8: Resonance stabilization of perthiyl radical and nitroxide radical.

The relative stability of RSS - versus RS. can also be deduced from the S-H bond dissociation energy (BDE) for RSS-H (approximately $70 \mathrm{kcal} / \mathrm{mol}$ ) [40] versus RS-H (approximately 92 $\mathrm{kcal} / \mathrm{mol}$ ) [42] indicating the increased relative stability of RSS. versus RS. Theoretical assessment of the reaction of TEMPOL with the model alkyl hydropersulfide, $\mathrm{CH}_{3} \mathrm{SSH}$, to give TEMPOL-OH and $\mathrm{CH}_{3} \mathrm{SS}$. indicates that the reaction is approximately $4.7 \mathrm{kcal} / \mathrm{mol}$ uphill in energy. This calculated value is in line with the reported O-H BDE for dialkylhydroxylamines (72 $\mathrm{kcal} / \mathrm{mol}$ for the $\mathrm{O}-\mathrm{H}$ bond in hydroxylamines [41] versus $70 \mathrm{kcal} / \mathrm{mol}$ for the $\mathrm{S}-\mathrm{H}$ bond in 
hydropersulfides) as being only slightly higher than that of the S-H BDE in RSS-H. The fact that the reaction of TEMPOL with RSSH is slightly uphill (but can be driven by RSS. dimerization in a purely chemical system) would predict that oxidation of isolated protein persulfides using TEMPOL (or a similar nitroxide) may not be efficient since perthiyl dimerization will be hindered. In this case, a slightly more oxidizing species may be required. Regardless, it is clear that oneelectron oxidation of RSSH is facile and can be accomplished by relatively weak oxidants.

It is important to note that $\mathrm{RSSH}$ is more acidic compared to the corresponding $\mathrm{RSH}$ by 1-2 $\mathrm{pK}_{\mathrm{a}}$ units. Thus, a higher percentage of the anionic RSS species exists compared to an $\mathrm{RS}^{-}$species. It can be argued that the observation that persulfides are superior reductants compared to thiols is simply a result of a greater percentage of the more reducing deprotonated species in the case of persulfides. Clearly, this can be a significant factor in cases where the anion is the reductant (as would be the case for electron transfer processes). However, it is thought that TEMPOL-mediated oxidations occur via $\mathrm{H}$-atom abstraction [43]. Therefore, in the case of nitroxide-mediated oxidations, RSSH are clearly superior reductants even though a smaller percentage of the protonated species exists compared to the corresponding $\mathrm{RSH}$. Moreover, in the reactions examined herein reduction by thiol was significantly less (or nearly nonexistent) under conditions where persulfides readily reduced oxidants. Thus, although it is

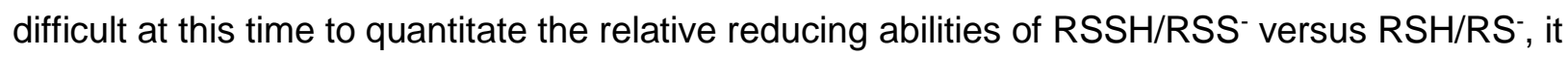
appears that RSSH/RSS- is clearly more reducing under most conditions.

It is well known that RS. reacts readily with $\mathrm{O}_{2}$ to give a number of sulfur oxides [for example, 44,45]. However, the results presented herein indicate a lack of reactivity between RSS - and $\mathrm{O}_{2}$. The lack of reactivity of RSS - with $\mathrm{O}_{2}$ is consistent with the finding that a solution generating MCP-SS. did not consume $\mathrm{O}_{2}$ (Figure 6a). Furthermore, computational evaluation indicates that formation of the MCP-OO. species is an uphill reaction, by approximately 16 $\mathrm{kcal} / \mathrm{mol}$ (Figure 6b). Of course, this calculated endergonicity can be overcome by subsequent favorable reactions of a fleeting MCP-OO- species thus driving this process. However, the lack of $\mathrm{O}_{2}$ consumption appears to indicate that this does not happen under the conditions of these experiments.

Consistent with our results, a recent report from the Pratt lab also finds that perthiyl radical species do not react with $\mathrm{O}_{2}$ [46]. In this study, photochemically induced homolysis of a tetrasulfide, RSSSSR, transiently generates RSS (monitored by the absorbance at $375 \mathrm{~nm}$ ), which then rapidly recombines back to the tetrasulfide $\left(k=6 \times 10^{9} \mathrm{M}^{-1} \mathrm{~s}^{-1}\right)$. Decay of the RSS. species was the same in both the presence and absence of $\mathrm{O}_{2}$, indicating a lack of $\mathrm{O}_{2}$ reactivity by RSS. 
In contrast to the results presented here and those from the Pratt lab [46], Everett and coworkers report that RSS. readily reacts with $\mathrm{O}_{2}$ with a reported second order rate constant of $5.1 \times 10^{6} \mathrm{M}^{-1} \mathrm{~s}^{-1}$ [37]. It may be important to note that the mechanism of generation of an RSS. species in the Everett study was via pulse radiolytic reduction of a dialkyl trisulfide, RSSSR, to give an RSSSR - species that presumably degrades rapidly to RSS- (measured by a transient absorbance at $374 \mathrm{~nm}$ ) and RS. In the current study, formation of the RSS. species is via direct oxidation of a hydropersulfide by obligate one-electron oxidants (e.g. TEMPOL, $\mathrm{K}_{3} \mathrm{Fe}(\mathrm{CN})_{6}$ and $\left.\mathrm{MbFe} \mathrm{e}^{\prime \prime \prime}\right)$. The exact reasons for these conflicting results are currently unknown and under investigation. However, it is possible that the previous pulse radiolysis study showing $\mathrm{O}_{2}$ consumption may have been a result of reaction with a fleeting RSSSR" species and not RSS. Alternatively, depending on the conditions of the experiments, the rapid RSS coupling reaction $\left(\mathrm{k}=6 \times 10^{9} \mathrm{M}^{-1} \mathrm{~s}^{-1}\right.$ ) may simply out-compete reaction with $\mathrm{O}_{2}$ (which is over $1000 \mathrm{x}$ slower, $\mathrm{k}=5.1 \times 10^{6} \mathrm{M}^{-1} \mathrm{~s}^{-1}$ ) although the coupling reaction is second order in RSS.

It is also known that RS. species react with NO to give S-nitrosothiols (RS-NO) [47]. However, similar to observations with $\mathrm{O}_{2}$, a solution generating RSS. (i.e. MCP-SS.) did not consume NO (Figure 7a). The expected product from the reaction of RSS. with NO would be the S-nitrosopersulfide (RSS-NO) (Reaction 7).

$$
\text { RSS. + NO } \rightarrow \text { RSS-NO }
$$

The inability of RSS to trap NO is likely to be due to the inherent weakness of the S-NO bond since the products from bond scission, NO and RSS., are relatively stable radical species. Thus, it would be predicted that generation of an RSS-NO compound via another distinct mechanism (besides RSS./NO radical coupling, Reaction 7) would also give NO and RSS. as the ultimate products. This was found to be the case as treatment of RSSH with an Snitrosothiol (which should transnitrosate to generate, initially, RSS-NO, Reaction 5) resulted in NO release via presumed homolytic cleavage of the incipient RSS-NO species, Reaction 6 (Figure 7b). Finally, computational analysis of this reaction indicates an extremely weak yet favorable interaction between RSS. and NO of approximately 3-4 kcal $/ \mathrm{mol}$ (Figure 7c). Since the dissociation reaction involves homolytic cleavage (Reaction 6), the S-NO BDE can be approximated to be $3-4 \mathrm{kcal} / \mathrm{mol}$. For comparison, the N-N bond in the NO dimer (ON-NO) is only slightly less (approximately $2 \mathrm{kcal} / \mathrm{mol}$ ) [for example, 48] consistent with the idea that the RSS-NO bond is too weak to exist at room temperature and above (akin to NO). Also, in both cases of homolysis, RSS-NO and ON-NO, entropy will favor bond dissociation. It is important to 
note that the S-NO BDE for an S-nitrosothiol, RS-NO, is significantly higher $(31-32 \mathrm{kcal} / \mathrm{mol}$ [49]) consistent with a lack of thermal dissociation of NO from $S$-nitrosothiols under normal physiological conditions.

The finding that an RSS-NO species is not stable (due to the extremely weak S-NO bond) may appear to be in conflict with recent reports indicating the stability and biological relevance associated with a similar species, - SSNO [for example, 50,51]. In order to address this issue, computational analysis of RSSNO and -SSNO reveals a comparatively shortened (and therefore stronger) S-N bond in the case of SSNO (Figure 9). This is consistent with a recent theoretical assessment of the structures of the protonated (HSSNO) versus anionic SSNO [52]. The shortening of the S-N bond in the anion is presumably due to an important resonance form that puts a positive charge adjacent to the anionic charge on the terminal sulfur atom of -SSNO, therefore giving partial double bond character to the S-N bond (Figure 9). Thus, it appears that our finding that RSS-NO species are inherently unstable with respect to homolytic cleavage to the corresponding perthiyl radical and NO is not necessarily in conflict with the idea that SSNO is more stable and may possess potential biological relevance. To go along with the shortened bond length for -SS-NO versus RSS-NO, the BDE for the S-N bond in SS-NO was calculated to be significantly higher (approximately $14 \mathrm{kcal} / \mathrm{mol}$ in the gas phase) compared to the S-N bond in the S-nitroso alkylpersulfide.
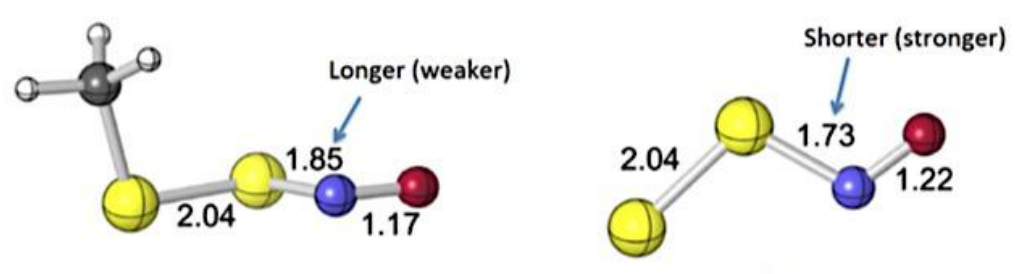

$\mathrm{CH}_{3} \mathrm{SSNO}$

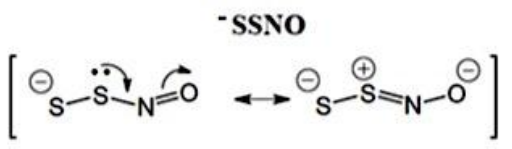

Figure 9: Computational analysis of $\mathrm{CH}_{3} \mathrm{SS}-\mathrm{NO}$ and ${ }^{-} \mathrm{SSNO}$.

As discussed previously, the reducing ability of RSSH/RSS- is, to a significant extent, due to the resonance stability of the perthiyl radical, RSS., an affect that also explains the stability of nitroxides (e.g. TEMPOL) (Figure 8). Thus the RSSH/RSS. and $\mathrm{R}_{2} \mathrm{NOH} / \mathrm{R}_{2} \mathrm{NO}$. redox couples can be viewed as being somewhat analogous. The redox biochemistry of 
nitroxides (including TEMPOL) consists of two accessible redox events, a redox couple between a hydroxylamine, $\mathrm{R}_{2} \mathrm{NOH}$, and a nitroxide, $\mathrm{R}_{2} \mathrm{NO}$ - (Reaction 8) and an additional redox couple between a nitroxide and an oxoammonium cation, $\mathrm{R}_{2} \mathrm{NO}^{+}$(Reaction 9).

$$
\begin{aligned}
& \mathrm{R}_{2} \mathrm{NOH} \rightleftharpoons \mathrm{R}_{2} \mathrm{NO} \cdot+\mathrm{e}^{-}+\mathrm{H}^{+-} \\
& \mathrm{R}_{2} \mathrm{NO} \cdot \rightleftharpoons \mathrm{R}_{2} \mathrm{NO}^{+}+\mathrm{e}^{-}
\end{aligned}
$$

Interestingly, TEMPOL has been shown to possess superoxide dismutase (SOD) activity [for example, 53] via cycling between the nitroxide and the oxoammonium cation [54] (Reactions 10 and 11).

$$
\begin{gathered}
\mathrm{R}_{2} \mathrm{NO}^{+}+\mathrm{O}_{2}^{-} \rightarrow \mathrm{R}_{2} \mathrm{NO} \cdot+\mathrm{O}_{2} \\
\mathrm{R}_{2} \mathrm{NO} \cdot+\mathrm{O}_{2}^{-}+2 \mathrm{H}^{+} \rightarrow \mathrm{R}_{2} \mathrm{NO}^{+}+\mathrm{H}_{2} \mathrm{O}_{2}
\end{gathered}
$$

The similarities between the persulfide and hydroxylamine systems necessitate the evaluation of a possible redox event involving oxidation of the perthiyl radical to a persulfyl cation (RSS ${ }^{+}$) species (Reaction 12).

$$
\text { RSS } \rightleftharpoons \mathrm{RSS}^{+}+\mathrm{e}^{-}
$$

Computational evaluation of the oxidation of $\mathrm{CH}_{3} \mathrm{SS}$. to $\mathrm{CH}_{3} \mathrm{SS}^{+}$indicates that the cation lies approximately $208 \mathrm{kcal} / \mathrm{mol}$ uphill in energy from the RSS - species, indicating that it is likely to be biologically inaccessible, unlike the corresponding oxidation of a nitroxide.

It should be noted that most of the studies herein were carried out using model donor species (e.g. MCPD) or simplified alkyl persulfides (computational work). Clearly, in the case of protein thiols, the protein environment can greatly influence their chemical biology [55]. Therefore, the fate and reactivity of individual protein persulfides will likely be a function of the nature of their protein environment. Regardless, the results presented herein provide evidence supporting the possible generation of stable biological perthiyls as one possible fate of a protein persulfide. Taken altogether it appears that hydropersulfides are good reductants capable of being oxidized by relatively weak oxidants. Moreover, the oxidized perthiyl radical species is stable in the presence of $\mathrm{O}_{2}$ and $\mathrm{NO}$ and only susceptible to dimerization to the tetrasulfide 
under the conditions of our experiments. The prevalence of hydropersulfides in cells, tissues, plasma and proteins [15] along with their susceptibility to oxidation, alludes to the strong possibility that perthiyl radicals will be formed in biological systems. In a biological setting, where perthiyl dimerization is not likely (due to the likelihood that they will either be at low levels or protected in proteins), the question remains as to their possible biological function, if any. The results presented herein indicate an accessible RSSH/RSS. (or RSS/RSS.) redox couple with both redox partners being fairly stable to, for example, reaction with $\mathrm{O}_{2}$ or NO. Although purely speculative at this time, it is intriguing to consider that cellular oxidative stress, conditions likely conducive to hydropersulfide formation [22], can generate persulfides in proteins, allowing them to then participate in a redox or electron transfer process. That is, a protein thiol may not possess the proper redox properties to participate in an electron transfer or redox event, but conversion to the corresponding persulfide may act as a "redox gate" allowing such chemistry to occur (Figure 10). Thus, only under oxidative stress will this system be activated (after all, persulfides are oxidized species that are more reducing than the thiols they are made from). Regardless, the findings presented here indicating the accessible and stable redox properties of persulfides can serve as the basis for further studies regarding their possible biological relevance and function.
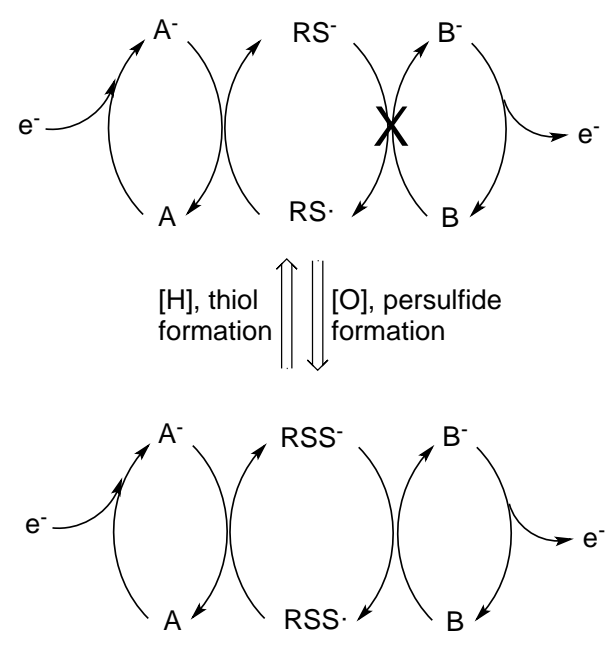

Figure 10: Possible role of protein RSSH formation for promoting redox biochemistry.

\section{Acknowledgments}

JMF wishes to acknowledge support from the National Institutes of Health (NIH) (HL106598) and the National Science Foundation (NSF) (CHE-1148641. JPT wishes to 
acknowledge support from the NSF (CHE-1213438 and CHE-1566065) and DJT wishes to acknowledge support from the NSF (CHE-030089) and the American Chemical Society Petroleum Research Fund (52801-ND4). The authors thank Prof. Joseph Lin (Sonoma State University), Prof. Peter Ford and Zhi Li (UCSB) for helpful discussions.

\section{References}

1. Kimura, H. (2014) The physiological role of hydrogen sulfide and beyond, Nitric Oxide, 41, 4-10.

2. Kimura, H. (2015) Physiological roles of hydrogen sulfide and polysulfides, Handb. Exp. Pharmacol., 230, 61-81.

3. Wallace, J. L. and Wang, R. (2015) Hydrogen sulfide-based therapeutics: Exploiting a unique but ubiquitous gasotransmitter, Nature Reviews, 14, 1-17.

4. Mani, S., Li, H., Untereiner, A., Wu, L., Yang, G., Austin, R. C., Dickout, J. G., Lhotak, S., Meng, Q. H., and Wang, R. (2013) Decreased endogenous production of hydrogen sulfide accelerates atherosclerosis, Circulation, 127, 2523-2534.

5. Hellmich, M. R. and Szabo, C. (2015) Hydrogen sulfide and cancer, Handb. Exp. Pharmacol., 230, 233-241.

6. Yang, G., Zhao, K., Ju, Y., Mani, S., Cao, Q., Puukila, S., Khaper, N., Wu, L. and Wang, R. (2012) Hydrogen sulfide protects against cellular senescence via S-sulfhydration of KEAP1 and activation of Nrf2, Antiox. Redox Signal., 18, 1906-1919.

7. Sen, N., Paul, B. D., Gadalla, M. M., Mustafa, A. K., Sen, T., Xu, R., Kim, S. and Snyder, S. H. (2013) Hydrogen sulfide-linked sulfhydration of NF- $\square$ B mediates its antiapoptotic actions, Mol. Cell, 45, 13-24.

8. Al-Magableh, M. and Hart, J. L. (2011) Mechanism of vasorelaxation and role of endogenous hydrogen sulfide production in mouse aorta, Naunyn-Schmied Arch. Pharmacol., 383, 403-413.

9. Bucci, M., Papapetropoulos, A., Vellecco, V., Zhou, Z., Pyriochou, A., Roussos, C., Roviezzo, F., Brancaleone, V. and Cirino, G. (2010) Hydrogen sulfide is an endogenous inhibitor of phosphodiesterase activity, Arterioscler. Thromb. Vasc. Biol., 30, 1998-2004.

10. Zhang, X. and Bian, J.-S. (2014) Hydrogen sulfide: A neuromodulator and neuroprotectant in the central nervous system, ACS Chem. Neurosci., 5, 876-883.

11. King, A. L. and Lefer, D. J. (2011) Cytoprotective actions of hydrogen sulfide in ischemia-reperfusion injury, Exp. Physiol., 96, 840-846.

12. Cavallini, D., Federici, G. and Barboni, E. (1970) Interactions of proteins with sulfide, Eur. J. Biochem., 14, 169-174. 
13. Francoleon, N. E., Carrington, S. J. and Fukuto, J. M. (2011) The reaction of $\mathrm{H}_{2} \mathrm{~S}$ with oxidized thiols: Generation of Persulfides and Implications to $\mathrm{H}_{2} \mathrm{~S}$ Biology, Arch. Biochem. Biophys., 516, 146-153.

14. Yamanishi, T. and Tuboi, S. (1981) The mechanism of the L-cystine cleavage reaction catalyzed by rat liver $\gamma$-cystathionase, J. Biochem., 89, 1913-1921.

15. Ida, T., Sawa, T., Ihara, H., Tsuchiya, Y., Watanabe, Y., Kumagai, Y., Suematsu, M., Motohashi, H., Fujii, S., MAtsunaga, T., Yamamoto, M., Ono, K., Devarie-Baez, N. O., Xian, M., Fukuto, J. M. and Akaike, T. (2014) Reactive cysteine persulfides and S-polythiolation regulate oxidative stress and redox signaling, Proc. Natl., Acad., Sci., USA, 111, 7606-7611.

16. Mustafa, A. K., Gadalla, M. M. and Snyder, S. H. (2009) Signaling by gasotransmitters, Science Signal., 2, 1-8.

17. Doka, A., Pader, I., Biro, A., Johansson, K., Chang, Q., Ballago, K., Prigge, J. R., PastorFlores, D., Dick, T. P., Schmidt, E. E., Arnar, E. S. J., and Nagy, P. (2016) A novel persulfide detection method reveals protein persulfide- and polysulfide-reducing functions of thioredoxin and glutathione systems. Sci. Adv., 2, e1500968.

18. Ono, K., Akaike, T., Sawa, T., Kumagai, Y., Wink, D. A., Tantillo, D. J., Hobbs, A. J., Nagy, P., Xian, M., Lin, J. and Fukuto, J. M. (2014) The redox chemistry and chemical biology of $\mathrm{H}_{2} \mathrm{~S}$, hydropersulfides and derived species: Implications of their possible biological activity and utility, Free Radic. Biol. Med., 77, 82-94.

19. Toohey, J. I. (1989) Sulphane sulphur in biological systems: A possible regulatory role, Biochem. J., 264, 625-632.

20. Pan J. and Carroll, K. S. (2013) Hydropersulfide reactivity in the detection of protein-S sulfhydration, ACS Chem .Biol., 8, 1110-1116.

21. Saund, S. S., Sosa, V., Henriquez, S., Nguyen, Q. N. N., Bianco, C. L., Soeda, S., Millikin, R., White, C., Le, H., Tantillo, D. J., Kumagai, Y., Akaike, T., Lin, J. and Fukuto, J. M. (2015) The chemical biology of hydropersulfides (RSSH): Chemical stability, reactivity and redox roles, Arch. Biochem. Biophys., 588, 15-24.

22. Millikin, R., Bianco, C. L., White, C., Saund, S. S., Henriquez, S., Sosa, V., Akaike, T., Kumagai, Y., Soeda, S., Toscano, J. P., Lin, J. and Fukuto, J. M. (2016) The chemical biology of protein hydropersulfides: Studies of a possible protective function of biological hydropersulfide generation, Free Rad. Biol. Med., 97, 136-147.

23. Artaud, I. and Galardon, E. (2014) A persulfide analogue of the nitrosothiol SNAP: Formation, characterization and reactivity, ChemBioChem, 15, 2361-2364.

24. Hart, T. W. (1985) Some observations concerning the S-nitroso and S-phenylsulfonyl derivatives of L-cysteine and glutathione, Tet. Lett., 26, 2013-2016.

25. Yordanov, N. D. and Ranguelova, K. (2000) Quantitative electron paramagnetic resonance and spectrophotometric determination of the free radical 4-hydroxy-2,2,6,6tetramethylpiperidinyoxy, Spectrochim. Acta Part A, 56, 373-378. 
26. Estabrook, R. W. (1961) Sudies of oxidative phosphorylation with potassium ferricyanide as electron acceptor, J. Biol. Chem., 236, 3015-3057.

27. Vasas, A., Dóka, É., Fábián, I. and Nagy, P. (2015) Kinetic and Thermodynamic Studies on the Disulfide Bond Reducing Potential of Hydrogen Sulfide. Nitric Oxide - Biol. Chem., 46, 93-101.

28. Hodgson, J.L., Namazian, M., Bottle, S. E. and Coote, M. L. (2007) One-electron oxidation and reduction potentials of nitroxide antioxidants: A theoretical study, J. Phys. Chem., 111, 13595-13605.

29. Miranda, K. M., Nims, R. W., Thomas, D. D., Espey, M. G., Citrin, D., Bartberger, M. D., Paolocci, N., Fukuto, J. M., Feelisch, M. and Wink, D. A. (2003) Comparison of the reactivity of nitric oxide and nitroxyl with heme proteins: A chemical discussion of the differential biological effects of these redox related products of NOS, J. Inorg. Biochem., 93, 52-60.

30. Marenich, A. V., Cramer, C. J. and Truhlar, D. G. (2009) Universal solvation model based on solute electron density and a continuum model of the solvent defined by the bulk dielectric constant and atomic surface tensions, J. Phys. Chem. B, 113, 6378-96.

31. Zhao, Y. and Truhlar, D. G. (2008) The M06 suite of density functionals for main group thermochemistry, thermochemical kinetics, noncovalent interactions, excited states, and transition elements: two new functionals and systematic testing of four M06-class functionals and 12 other functionals, Theor. Chem. Acc., 120, 215-41.

32. Gaussian 09, Revision D; Gaussian, Inc.: Wallingford, CT, 2009.

33. Fukui, K. (1981) "The path of chemical-reactions - The IRC approach," Acc. Chem. Res., 14, 363-68.

34. Hratchian, H. P. and Schlegel, H. B. in Theory and Applications of Computational Chemistry: The First 40 Years, Ed. C. E. Dykstra, G. Frenking, K. S. Kim, and G. Scuseria (Elsevier, Amsterdam, 2005) 195-249.

35. Zhao, Y., Bhushan, S., Yang, C., Otsuka, H., Stein, J. D., Pacheco, A., Peng, B., Devarie-Baez, N. O., Aguilar, H. C., Lefer, D. J. and Xian, M. (2013) Controllable hydrogen sulfide donors and their activity against myocardial ischemia-reperfusion injury, ACS Chem. Biol., 8, 1283-1290

36. Chakrabarti, M. H. and Roberts, E. P. L. (2008) Analysis of mixtures of ferrocyanide and ferricyanide using UV-visible spectroscopy for characterization of a novel redox flow battery, $J$. Chem. Soc. Pak., 30, 817-823.

37. Everett, S. A., Schoneich, C., Stewart, J. H. and Asmus, K.-D. (1992) Perthiyl radicals, trisulfide radical ions, and sulfate formation. A combined photolysis and radiolysis study on redox processes with organic di- and trisulfides. J. Phys. Chem., 96, 306-314.

38. Williams, D. L. H. (1999) The chemistry of S-nitrosothiols, Acc. Chem. Res., 32, 869876. 
39. Everett, S.A., Folkes, L. K., Wardman, P. and Asmus, K.-D. (1994) Free-radical repair by a novel perthiol: Reversible hydrogen transfer and perthiyl radical formation, Free Rad. Res., 20, 387-400.

40. Everett, S. A. and Wardman, P. (1995) Perthiols as antioxidants: Radical-scavenging and prooxidative mechanisms, Meth. Enzymol., 251, 55-69.

41. Mahoney, L. R., Mendenhall, G. D. and Ingold, K. U. (1973) Calorimetric and equilibrium studies on some stable nitroxide and iminoxyl radicals. Approximate $\mathrm{O}-\mathrm{H}$ bond dissociation energies in hydroxylamines and oximes, J. Am. Chem. Soc., 95, 8610-8614.

42. Benson, S.W. (1978) Thermochemistry and kinetics of sulfur-containing molecules and radicals, Chem. Rev., 78, 23-35.

43. Wertz, S. and Studer, A. (2013) Nitroxide-catalyzed transition-metal-free aerobic oxidation processes, Green Chem., 15, 3116-3134.

44. Schafer, K., Bonifacic, M., Bahnemann, D. and Asmus, K.-D. (1978) Addition of oxygen to sulfur radicals, J. Phys. Chem., 82, 2777-2780.

45. Sevilla, M. D., Becker, D., Swarts, S. and Herrington, J. (1987) Sulfinyl radical formation from the reaction of cysteine and glutathione thiyl radicals with molecular oxygen, Biochem. Biophys. Res. Commun., 144, 1037-1042.

46. Chauvin, J-P. R., Haidasz, E. A., Griesser, M. and Pratt, D. A. (2016) Polysulfide-1oxides react with peroxyl radicals as quickly as hindered phenolic antioxidants and do so by a surprising concerted homolytic substitution, Chem. Sci., DOI: 10.1039/c6sc01434h.

47. Madej, E., Folkes, L. K., Wardman, P., Czapski, G. and Goldstein, S. (2008) Thiyl radicals react with nitric oxide to form $S$-nitrosothiols with rates constants near diffusioncontrolled limit, Free Radic. Biol. Med., 44, 2013-2018.

48. Brookes, M. D., McKellar, A. R. W. and Amano, T. (1997) Millimeter-wave spectrum of the NO-dimer, J. Mol. Spectrosc., 185, 153-157.

49. Bartberger, M. D., Mannion, J. D., Powell, S. C., Stamler, J. S., Houk, K. N. and Toone, E. J. (2001) S-N Dissociation energies of S-nitrosothiols: On the origins of S-nitrosothiol decomposition rates, J. Am. Chem. Soc., 123, 8868-8869.

50. Cortese-Krott, M. M., Fernandez, B. O., Santos, J. L. T., Mergia, E., Grman, M., Nagy, P., Kelm, M., Butler, A. and Feelisch, M. (2014) Nitrosopersulfide (SSNO) accounts for sustained NO bioactivity of S-nitrosothiols following reaction with sulfide, Redox Biol., 2, 234244.

51. Cortese-Krott, M. M., Kuhnle, G. G. C., Dyson, A., Fernandez, B. O., Grman, M., DuMond, J. F., Barrow, M. P., McLeod, G., Nakagawa, H., Ondrias, K., Nagy, P., King, S. B., Saavedra, J. E., Keefer, L. K., Singer, M., Kelm, M., Butler, A. R., and Feelisch, M. (2015) Key bioactive reaction products of the $\mathrm{NO} / \mathrm{H}_{2} \mathrm{~S}$ interaction are $\mathrm{S} / \mathrm{N}$-hybrid species, polysulfides, and nitroxyl, Proc. Natl. Acad. Sci., USA, 112, E4651-E4660. 
52. Wedmann, R., Zahl, A., Shubina, T. E., Durr, M., Heinemann, F. W., Bugenhagen, B. E. C., Burger, P., Ivanovic-Burmazovic, I. and Filipovic, M. R. (2015) Does perthionitrite (SSNO-) account for sustained bioactivity of NO? A (bio)chemical characterization, Inorg. Chem., 54, 9367-9380.

53. Samuni, A., Mitchell, J. B., DeGraff, W., Krishna, C. M., Samuni, U. and Russo, A., (1991) Nitroxide SOD-mimics: Modes of action, Free Rad. Res. Commun., 12-13, 187-194.

54. Goldstein, S., Merenyi, G., Russo, A. and Samuni, A. (2002) The role of oxoammonium cation in the SOD-mimic activity of cyclic nitroxides, J. Am. Chem. Soc., 125, 789-795.

55. Go, Y.-M., Chandler, J. and Jones, D. P. (2015) The cysteine proteome, Free Radic. Biol. Med., 84, 227-245. 